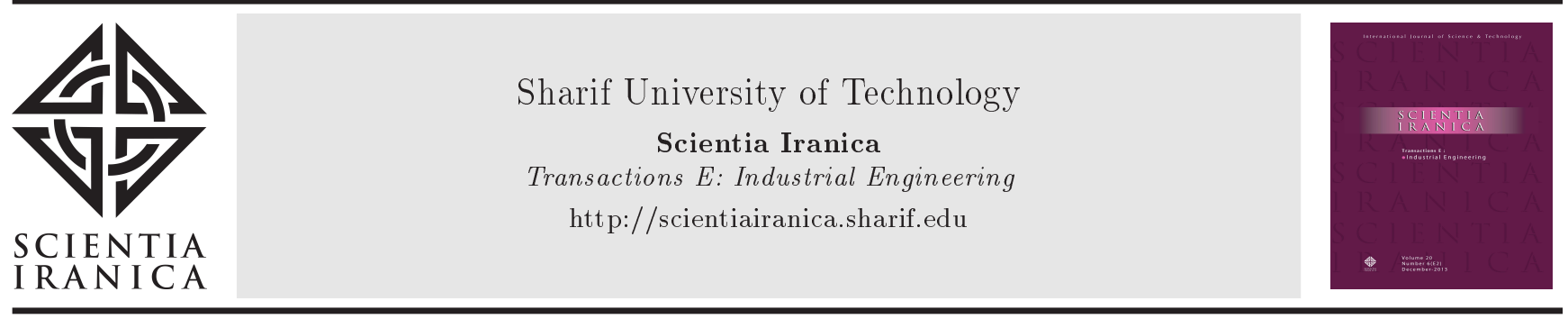

\title{
Prioritized averaging/geometric aggregation operators under the intuitionistic fuzzy soft set environment
}

\author{
R. Arora and H. Garg* \\ School of Mathematics, Thapar Institute of Engineering \& Technology (Deemed University), Patiala 147004, Punjab, India.
}

Received 22 June 2016; received in revised form 8 September 2016; accepted 10 December 2016

KEYWORDS
MCDM;
IFSS;
Aggregation operator;
Decision-making.

\section{Introduction}

Multiple Criteria Group Decision Making Problems (MCGDM) are important parts of modern decision theory due to rapid development of economic and social uncertainties. Today, Decision-Maker (DM) wants to attain more than one goal by satisfying different constraints. But, due to the complexity of management environments and decision problems themselves, DMs may provide their rating or judgment in the form of crisp numbers without considering the degree of fuzziness or vagueness of the data in the domain of the problem [1]. However, in these days, uncertainties play a dominant role during the decision-making process, and the decision-maker cannot give their preferences to an accurate level without being proper handled. The main objective during an analysis is to handle the proper data so as to minimize the uncertainties level. To handle this, a fuzzy set theory [2] has been

*. Corresponding author. Tel.: +918699031147 E-mail address: harishg58iitr@gmail.com (H. Garg)

doi: $10.24200 /$ sci.2017.4410 successfully applied. Having observed its successful implementation, researchers have extended their theory to the Intuitionistic Fuzzy Set (IFS) [3] and IntervalValued Intuitionistic Fuzzy Set (IVIFS) [4] so as to minimize the uncertainty level. After their successful extensions, various researchers have applied it to the decision-making process. For instance, $\mathrm{Xu}$ [5], $\mathrm{Xu}$ and Yager [6] developed weighted averaging and geometric aggregation operators under IFS environment. Wang and Liu $[7,8]$ investigated these aggregation operators by using Einstein operations. Garg [9] presented a generalized intuitionistic fuzzy interactive geometric aggregation operators using Einstein t-norm and tconorm operations. Garg et al. [10] presented an entropy-based approach to solving the decision-making problem under fuzzy environment. Garg [11] developed a new generalized improved score function to rank the IVIFSs. Verma and Sharma [12] presented an intuitionistic fuzzy prioritized weighted average operator under the Einstein norm operations. $\mathrm{Xu}$ and Chen [13], and $\mathrm{Xu}$ [14] developed some arithmetic and geometric aggregation operators, namely intervalvalued intuitionistic fuzzy weighted averaging and geometric operators, for aggregating the interval-valued 
intuitionistic fuzzy information. Apart from that, various researchers pay more attention to decisionmaking process to aggregate different alternatives using different aggregation operators [15-23] and their corresponding references.

Since the above theories have been successfully applied in various disciplines, they have certain limitations; for instance, their theories are restricted to their parametrization tools, and hence they cannot be applied effectively to real life problems. To handle these, soft set theory [24] pays a great deal of attention and successfully copes with these types of conditions. After that, many authors have shown an intense interest in the matter $[25,26]$. Maji et al. [27,28] combined the theories of soft set with the fuzzy and intuitionistic fuzzy set and came up with a new concept of Fuzzy Soft Set (FSS) and Intuitionistic Fuzzy Soft Set (IFSS). The advantages of these extended theories are that they are capable of facilitating the descriptions of the realworld situation with the help of their parameterizations property. Meanwhile, the study of hybrid model, which combines the soft sets with other mathematical structure, also pays a great deal of attention; hence, an active research topic of soft set theory will be presented. A lot of extensions of soft set model have also been developed recently on intuitionistic fuzzy soft sets [27,29], generalized fuzzy soft set [30,31], generalized intuitionistic fuzzy soft set $[32,33]$, distance measures [34-38], and fuzzy number intuitionistic fuzzy soft sets [39]. Apart from that, FSSs have been also successfully applied to MCDM problems in recent years $[40,41]$.

It has been concluded that these existing approaches work well under restriction in which the parameters and decision-makers are at same priority level. But, this assumption needs to be relaxed to analyze decisions better. Furthermore, up to now, the research on FSS and IFSS is only about their basic theory and applications, but there is no research on the information aggregation fusion. So, to investigate this issue, the present work proposed some prioritized aggregation operations under IFSSs environment by taking advantage of the prioritized aggregation operator [42]. Thus, by considering the fact that the IFSS has a powerful tool to deal with the ambiguity and vagueness of the data, the work introduced a series of aggregation operators, namely the intuitionistic fuzzy soft prioritized weighted and ordered weighted averaging, intuitionistic fuzzy soft prioritized weighted, and ordered weighted geometric. Furthermore, these operators have been tested on the problem of MCDM, where the most desirable alternative has been computed under the set of different criteria. Finally, the computed results are compared with that of the existing operators to show their effectiveness.

This paper is organized as follows. Section 2 describes the overview of SS, FSS, and IFSS theories. Section 3 defines the operational laws on IFSS. Section 4 presents the averaging/geometric priorities aggregated operators, namely IFSPWA, IFSPOWA, IFSPWG, IFSPOWG along with their properties. Section 5 describes the MCDM approach under IFSS environment and demonstrates it with the help of an illustrative example. Finally, a concrete conclusion is summarized in Section 6.

\section{Preliminaries}

Some basic definitions related to SS, FSS, IFSS are reviewed on universal set $U$.

Definition 2.1. Soft sets [24]: Let $E$ be the set of parameters. A pair $(F, E)$ is called soft set (SS) over $U$ where $F: E \rightarrow K^{U}$, the set of all subsets of $U$.

Example 2.1. Consider a set of four houses given by $U=\left\{h_{1}, h_{2}, h_{3}, h_{4}\right\}$ and $E=$ \{expensive " $\left(e_{1}\right)$ ", "wooden $\left(e_{2}\right)$ ", "cheap $\left(e_{3}\right)$ ", "beautiful $\left(e_{4}\right)$ ", "in good location $\left(e_{5}\right)$ " $\}$ be a set of parameters. If $F: E \rightarrow K^{U}$ be defined by $F_{e_{1}}=\left\{h_{2}, h_{3}\right\}$, $F_{e_{2}}=\left\{h_{1}, h_{3}, h_{4}\right\}, F_{e_{3}}=\left\{h_{1}, h_{4}\right\}, F_{e_{4}}=\left\{h_{1}, h_{2}, h_{3}\right\}$, $F_{e_{5}}=\left\{h_{2}, h_{4}\right\}$, then the soft set, which describes the houses, is defined as $(F, E)=\left\langle F_{e_{1}}, F_{e_{2}}, F_{e_{3}}, F_{e_{4}}, F_{e_{5}}\right\rangle$.

Definition 2.2. Fuzzy Soft Sets (FSS) [43]: Let $I^{U}$ denote the set of all fuzzy subsets of $U$, and then a pair $(F, E)$ is called FSS over $U$ if $F$ is a mapping derived from $E$ to $I^{U}$ and is defined as $F_{e_{j}}=\left\{\left\langle x, \mu_{j}(x)\right\rangle \mid x \in\right.$ $U\}$. For any parameter $e_{j}$, FSS reduces to SS if $F_{e_{j}}$ is a crisp subset of $U$.

Example 2.2. Consider Example 2.1 for describing the "attractiveness of the houses" then FSS corresponding to what has been defined as $F_{e_{1}}=$ $\left\{\left\langle\left(h_{2}, 0.2\right),\left(h_{3}, 0.7\right)\right\rangle\right\}, F_{e_{2}}=\left\{\left\langle\left(h_{1}, 0.6\right),\left(h_{3}, 0.7\right),\left(h_{4}\right.\right.\right.$, $0.9)\rangle\}, F_{e_{3}}=\left\{\left\langle\left(h_{1}, 0.3\right),\left(h_{4}, 0.5\right)\right\rangle\right\}, F_{e_{4}}=\left\{\left\langle\left(h_{1}, 0.6\right)\right.\right.$, $\left.\left.\left(h_{2}, 0.9\right),\left(h_{3}, 0.7\right)\right\rangle\right\}$ and $F_{e_{5}}=\left\{\left\langle\left(h_{2}, 0.7\right),\left(h_{4}, 0.6\right)\right\}\right.$.

Definition 2.3. Intuitionistic Fuzzy Soft Sets (IFSS) [27]: Let IFS $(U)$ denote the set of all intuitionistic fuzzy subsets of $U$, then a pair $(F, E)$ is called an IFSS over $U$ iff $F: E \rightarrow \operatorname{IFS}(U)$, such that for any parameter $e_{j} \in E, F_{e_{j}}$ can be written as follows:

$$
F_{e_{j}}\left(x_{i}\right)=\left\{\left\langle x_{i}, \mu_{j}\left(x_{i}\right), \nu_{j}\left(x_{i}\right)\right\rangle \mid x_{i} \in U\right\},
$$

where $\mu_{j}\left(x_{i}\right)$ and $\nu_{j}\left(x_{i}\right)$ are degrees of membership and non-membership, respectively, with conditions $0 \leq$ $\mu_{j}\left(x_{i}\right), \nu_{j}\left(x_{i}\right) \leq 1$ and $\mu_{j}\left(x_{i}\right)+\nu_{j}\left(x_{i}\right) \leq 1$.

For the sake of simplicity, we denote this pair to be $F_{e_{i j}}=\left\langle\mu_{i j}, \nu_{i j}\right\rangle$ and called as an Intuitionistic Fuzzy Soft Number (IFSN). 
Table 1. Representation of IFSS for describing the attractiveness of the houses.

\begin{tabular}{cccccc}
\hline $\boldsymbol{F}$ & $\boldsymbol{e}_{\boldsymbol{1}}$ & $\boldsymbol{e}_{\mathbf{2}}$ & $\boldsymbol{e}_{\mathbf{3}}$ & $\boldsymbol{e}_{\mathbf{4}}$ & $\boldsymbol{e}_{\mathbf{5}}$ \\
\hline$h_{1}$ & $\langle 0.2,0.6\rangle$ & $\langle 0.1,0.5\rangle$ & $\langle 0.9,0.1\rangle$ & $\langle 0.3,0.4\rangle$ & $\langle 0.6,0.2\rangle$ \\
$h_{2}$ & $\langle 0.5,0.4\rangle$ & $\langle 0.2,0.5\rangle$ & $\langle 0.3,0.7\rangle$ & $\langle 0.6,0.2\rangle$ & $\langle 0.4,0.4\rangle$ \\
$h_{3}$ & $\langle 0.6,0.2\rangle$ & $\langle 0.5,0.4\rangle$ & $\langle 0.8,0.1\rangle$ & $\langle 0.6,0.4\rangle$ & $\langle 0.2,0.7\rangle$ \\
$h_{4}$ & $\langle 0.4,0.5\rangle$ & $\langle 0.3,0.5\rangle$ & $\langle 0.6,0.1\rangle$ & $\langle 0.4,0.1\rangle$ & $\langle 0.2,0.4\rangle$ \\
\hline
\end{tabular}

Example 2.3. Consider the description of the houses as given in Example 2.1. Then, the rating values of each house for a particular parameter are represented in terms of IFSS given in Table 1.

In the process of applying IFSNs to practical problems, it is necessary to rank them. Moreover, a score function of $F_{e_{i j}}$ is defined as follows:

$$
S\left(F_{e_{i j}}\right)=\frac{1+\mu_{i j}-\nu_{i j}}{2},
$$

where $S\left(F_{e_{i j}}\right) \in[0,1]$. From this definition, it has been seen that the larger $S\left(F_{e_{i j}}\right)$ is, the larger IFSN $F_{e_{i j}}$ will be.

Example 2.4. Let $F_{e_{11}}=\langle 0.4,0.2\rangle$ and $F_{e_{12}}=$ $\langle 0.3,0.5\rangle$ be two IFSNs, then by using Eq. (1), we get $S\left(F_{e_{11}}\right)=0.2$ and $S\left(F_{e_{12}}\right)=-0.2$. Since $S\left(F_{e_{11}}\right)>$ $\left(F_{e_{12}}\right)$, we have $F_{e_{11}}>F_{e_{12}}$.

However, in some situations, the above function cannot be used to compare IFSNs. For example, let $F_{e_{11}}=\langle 0.2,0.4\rangle$ and $F_{e_{12}}=\langle 0.3,0.5\rangle$, then it is impossible to know which one is bigger because $S\left(F_{e_{11}}\right)=S\left(F_{e_{12}}\right)$. For this, accuracy function $H$ of $F_{e_{i j}}$ is defined as follows:

$$
H\left(F_{e_{i j}}\right)=\mu_{i j}+\nu_{i j},
$$

where $H\left(F_{e_{i j}}\right) \in[0,1]$. Thus, in order to compare two IFSNs $F_{e_{i j}}$ and $G_{e_{i j}}$, the following ranking and comparison laws of two IFSNs are defined below:

1. If $S\left(F_{e_{i j}}\right)>S\left(G_{e_{i j}}\right)$, then $F_{e_{i j}}>G_{e_{i j}}$;

2. If $S\left(F_{e_{i j}}\right)=S\left(G_{e_{i j}}\right)$, then:

- If $H\left(F_{e_{i j}}\right)>H\left(G_{e_{i j}}\right)$, then $F_{e_{i j}}>G_{e_{i j}}$;

- If $H\left(F_{e_{i j}}\right)=H\left(G_{e_{i j}}\right)$, then $F_{e_{i j}}=G_{e_{i j}}$.

Definition 2.4. Prioritized Weighted Average (PWA) operator [42]: Let $A=\left\{A_{1}, A_{2}, \cdots, A_{n}\right\}$ be a collection of attributes and let there be a prioritization between the attributes expressed by the linear ordering $A_{1} \succ A_{2} \succ \cdots \succ A_{n}$, indicating that attribute $A_{j}$ has higher priority than $A_{k}$ if $j<k$. Also, let $A_{j}(x)$ be a performance value of option $x$ under attribute $A_{j}$, such that $A_{j}(x) \in[0,1]$. If:

$$
\operatorname{PWA}\left(A_{1}, A_{2}, \cdots, A_{n}\right)=\sum_{j=1}^{n} \frac{T_{j}}{\sum_{j=1}^{n} T_{j}} A_{j},
$$

where $T_{j}=\prod_{k=1}^{j-1} A_{k}, j=2,3, \cdots, n$, and $T_{1}=1$, then $\operatorname{PWA}\left(A_{1}, A_{2}, \cdots, A_{n}\right)$ is called the Prioritized Weighted Average (PWA) operator.

\section{Operational law for IFSNs}

In this section, we introduce some operations on IFSNs and solve some of their desirable properties.

Definition 3.1. Let $F_{e}=\langle\mu, \nu\rangle, F_{e_{11}}=\left\langle\mu_{11}, \nu_{11}\right\rangle$ and $F_{e_{12}}=\left\langle\mu_{12}, \nu_{12}\right\rangle$ be three IFSNs, and for any real number $\lambda>0$, by algebraic norms, we have the following equations:

(i) $F_{e_{11}} \oplus F_{e_{12}}=\left\langle\mu_{11}+\mu_{12}-\mu_{11} \mu_{12}, \nu_{11} \nu_{12}\right\rangle$;

(ii) $F_{e_{11}} \otimes F_{e_{12}}=\left\langle\mu_{11} \mu_{12}, \nu_{11}+\nu_{12}-\nu_{11} \nu_{12}\right\rangle$;

(iii) $\lambda F_{e}=\left\langle 1-(1-\mu)^{\lambda}, \nu^{\lambda}\right\rangle$;

(iv) $F_{e}^{\lambda}=\left\langle\mu^{\lambda}, 1-(1-\nu)^{\lambda}\right\rangle$.

Theorem 3.1. All the operation laws for IFSNs as given in Definition 3.1, i.e. $F_{e_{11}} \oplus F_{e_{12}}, F_{e_{11}} \otimes F_{e_{12}}, \lambda F_{e}$, and $F_{e}^{\lambda}$, are also IFSNs.

Proof. Since $F_{e_{1 j}}(j=1,2)$ is IFSNs, this implies $0 \leq$ $\mu_{1 j} \leq 1,0 \leq \nu_{1 j} \leq 1,0 \leq \mu_{1 j}+\nu_{1 j} \leq 1$, and hence $0 \leq$ $\left(1-\mu_{11}\right)\left(1-\mu_{12}\right) \leq 1 \Leftrightarrow 0 \leq 1-\left(1-\mu_{11}\right)\left(1-\mu_{12}\right) \leq 1$ and $0 \leq \nu_{11} \nu_{12} \leq 1$. Further, $1-\left(1-\mu_{11}\right)\left(1-\mu_{12}\right)+$ $\nu_{11} \nu_{12} \leq 1-\nu_{11} \nu_{12}+\nu_{11} \nu_{12} \leq 1$. Thus, $F_{e_{11}} \oplus F_{e_{12}}$ is IFSNs.

Also, $1-(1-\mu)^{\lambda} \geq 0, \nu^{\lambda} \geq 0,1-(1-\mu)^{\lambda}+\nu^{\lambda} \leq$ $1-(1-\mu)^{\lambda}+(1-\mu)^{\lambda} \leq 1$. Thus, $\lambda F_{e}$ is also an IFSN, which is true similarly for others.

Theorem 3.2. (Commutative law) Let $F_{e_{1 j}}=\left\langle\mu_{1 j}\right.$, $\left.\nu_{1 j}\right\rangle(j=1,2)$ be two IFSNs, then:

(i) $F_{e_{11}} \oplus F_{e_{12}}=F_{e_{12}} \oplus F_{e_{11}}$;

(ii) $F_{e_{11}} \otimes F_{e_{12}}=F_{e_{12}} \otimes F_{e_{11}}$.

Theorem 3.3. (Associative law) Let $F_{e_{1 j}}=\left\langle\mu_{1 j}, \nu_{1 j}\right.$ \rangle$(j=1,2,3)$ be three IFSNs, then:

(i) $\left(F_{e_{11}} \oplus F_{e_{12}}\right) \oplus F_{e_{13}}=F_{e_{11}} \oplus\left(F_{e_{12}} \oplus F_{e_{13}}\right)$;

(ii) $\left(F_{e_{11}} \otimes F_{e_{12}}\right) \otimes F_{e_{13}}=F_{e_{11}} \otimes\left(F_{e_{12}} \otimes F_{e_{13}}\right)$.

Theorems 3.2 and 3.3 are straightforward, so we omit their proofs.

Theorem 3.4. Let $F_{e}=\langle\mu, \nu\rangle$ and $F_{e_{1 j}}=\left\langle\mu_{1 j}, \nu_{1 j}\right\rangle$ $(j=1,2)$ be three IFSNs, and then for real numbers $\lambda$ 's we have:

(i) $\lambda\left(F_{e_{11}} \oplus F_{e_{12}}\right)=\lambda F_{e_{11}} \oplus \lambda F_{e_{11}}$;

(ii) $\left(F_{e_{11}} \otimes F_{e_{12}}\right)^{\lambda}=F_{e_{11}}^{\lambda} \otimes F_{e_{11}}^{\lambda}$;

(iii) $\lambda_{1} F_{e} \oplus \lambda_{2} F_{e}=\left(\lambda_{1}+\lambda_{2}\right) F_{e}$;

(iv) $F_{e}^{\lambda_{1}} \otimes F_{e}^{\lambda_{2}}=F_{e}^{\lambda_{1}+\lambda_{2}}$. 
Proof. We prove parts (i) and (iii), and hence do similarly for others.

(i) For real number $\lambda>0, \lambda F_{e_{11}}=\left\langle 1-\left(1-\mu_{11}\right)^{\lambda}, \nu_{11}^{\lambda}\right\rangle$ and $\lambda F_{e_{12}}=\left\langle 1-\left(1-\mu_{12}\right)^{\lambda}, \nu_{12}^{\lambda}\right\rangle$. Thus:

$$
\begin{aligned}
& \lambda F_{e_{11}} \oplus \lambda F_{e_{12}} \\
&=\left\langle 1-\left(1-\left(1-\left(1-\mu_{11}\right)^{\lambda}\right)\right)\right. \\
&\left.\left(1-\left(1-\left(1-\mu_{12}\right)^{\lambda}\right)\right), \nu_{11}^{\lambda} \nu_{12}^{\lambda}\right\rangle \\
&=\left\langle 1-\left(1-\mu_{11}\right)^{\lambda}\left(1-\mu_{12}\right)^{\lambda}, \nu_{11}^{\lambda} \nu_{12}^{\lambda}\right\rangle \\
&=\left\langle 1-\left(\left(1-\mu_{11}\right)\left(1-\mu_{12}\right)\right)^{\lambda},\left(\nu_{11} \nu_{12}\right)^{\lambda}\right\rangle \\
&= \lambda\left(F_{e_{11}} \oplus F_{e_{12}}\right) .
\end{aligned}
$$

Hence, the result.

(iii) For $\lambda_{1}, \lambda_{2}>0$ and IFSN $F_{e}=\langle\mu, \nu\rangle$, we have:

$$
\begin{aligned}
& \lambda_{1} F_{e}=\left\langle 1-(1-\mu)^{\lambda_{1}}, \nu^{\lambda_{1}}\right\rangle, \\
& \lambda_{2} F_{e}=\left\langle 1-(1-\mu)^{\lambda_{2}}, \nu^{\lambda_{2}}\right\rangle .
\end{aligned}
$$

Thus:

$$
\begin{aligned}
& \lambda_{1} F_{e} \oplus \lambda_{2} F_{e} \\
&=\left\langle 1-\left(1-\left(1-(1-\mu)^{\lambda_{1}}\right)\right)\right. \\
&\left.\left(1-\left(1-(1-\mu)^{\lambda_{2}}\right)\right), \nu^{\lambda_{1}} \nu^{\lambda_{2}}\right\rangle \\
&=\left\langle 1-(1-\mu)^{\lambda_{1}}(1-\mu)^{\lambda_{2}}, \nu^{\lambda_{1}} \nu^{\lambda_{2}}\right\rangle \\
&=\left\langle 1-(1-\mu)^{\lambda_{1}+\lambda_{2}}, \nu^{\lambda_{1}+\lambda_{2}}\right\rangle \\
&=\left(\lambda_{1}+\lambda_{2}\right) F_{e} .
\end{aligned}
$$

Hence, the result.

\section{Averaging/geometric prioritized aggregation operators}

In this section, we will investigate the Prioritized Aggregation (PA) averaging/geometric operators under IFSS environment.

\subsection{Intuitionistic Fuzzy Soft Prioritized Weighted Average (IFSPWA) operator}

In this section, we will introduce the weighted averaging PA operator named as IFSPWA operator for the collections of IFSNs.

Definition 4.1. Let $F_{e_{i j}}=\left\langle\mu_{i j}, \nu_{i j}\right\rangle,(i=1,2, \ldots$, $m ; j=1,2, \ldots, n)$ be collections of IFSNs. Then, we have:

$$
\begin{aligned}
\operatorname{IFSPWA}( & \left.F_{e_{11}}, F_{e_{12}}, \cdots, F_{e_{m n}}\right) \\
= & \bigoplus_{i=1}^{m} \frac{T_{i}}{\sum_{i=1}^{m} T_{i}}\left(\bigoplus_{j=1}^{n} \frac{R_{j}}{\sum_{j=1}^{n} R_{j}} F_{e_{i j}}\right)
\end{aligned}
$$

where $R_{1}=1, T_{1}=1$ and $R_{j}=\prod_{l=1}^{j-1} S\left(F_{e_{i l}}\right),(j=$ $2,3, \cdots, n), T_{i}=\prod_{k=1}^{i-1} S\left(F_{e_{k}}\right)(i=2,3, \cdots, m)$ where $S\left(F_{e_{i j}}\right)$ represents the score function of IFSN $F_{e_{i j}}$.

By using Definition 4.1, we can get the following result.

Theorem 4.1. The aggregated value of all IFSNs by IFSPWA operator is an IFSN defined as follows:

$$
\begin{aligned}
\operatorname{IFSPWA}( & \left.F_{e_{11}}, F_{e_{12}}, \cdots, F_{e_{m n}}\right) \\
= & \left\langle 1-\prod_{i=1}^{m}\left(\prod_{j=1}^{n}\left(1-\mu_{i j}\right)^{\frac{R_{j}}{\sum_{j=1}^{n} R_{j}}}\right)^{\frac{T_{i}}{\sum_{i=1}^{m} T_{i}}},\right. \\
& \left.\prod_{i=1}^{m}\left(\prod_{j=1}^{n} \nu_{i j}^{\frac{R_{j}}{\sum_{j=1}^{n} R_{j}}}\right)^{\frac{T_{i}}{\sum_{i=1}^{m} T_{i}}}\right\rangle .
\end{aligned}
$$

Proof. For $n=1$, we have:

$$
\begin{aligned}
\operatorname{IFSPWA} & \left(F_{e_{11}}, F_{e_{21}}, \ldots, F_{e_{m 1}}\right) \\
= & \bigoplus_{i=1}^{m} \frac{T_{i}}{\sum_{i=1}^{m} T_{i}} F_{e_{i 1}} \\
= & \left\langle 1-\prod_{i=1}^{m}\left(1-\mu_{i 1}\right)^{\frac{T_{i}}{\sum_{i=1}^{m} T_{i}}}, \prod_{i=1}^{m} \nu_{i 1}^{\frac{T_{i}}{\sum_{i=1}^{m} T_{i}}}\right\rangle \\
= & \left\langle 1-\prod_{i=1}^{m}\left(\prod_{j=1}^{1}\left(1-\mu_{i j}\right)^{\frac{R_{j}}{\sum_{j=1}^{1} R_{j}}}\right)^{\frac{T_{i}}{\sum_{i=1}^{m} T_{i}}},\right. \\
& \left.\prod_{i=1}^{m}\left(\prod_{j=1}^{1} \frac{R_{j}}{\sum_{i j}^{1}}\right)^{\frac{T_{i}}{\sum_{i=1}^{m} R_{i}}}\right\rangle,
\end{aligned}
$$

and for $m=1$ :

$$
\begin{aligned}
\operatorname{IFSPWA} & \left(F_{e_{11}}, F_{e_{12}}, \cdots, F_{e_{1 n}}\right) \\
& =\bigoplus_{j=1}^{n} \frac{R_{j}}{\sum_{j=1}^{n} R_{j}} F_{e_{1 j}} \\
& =\left\langle 1-\prod_{j=1}^{n}\left(1-\mu_{1 j}\right)^{\frac{R_{j}}{\sum_{j=1}^{n} R_{j}}}, \prod_{j=1}^{n} \nu_{1 j}^{\frac{R_{j}}{\sum_{j=1}^{n} R_{j}}}\right\rangle
\end{aligned}
$$




$$
\begin{gathered}
=\left\langle 1-\prod_{i=1}^{1}\left(\prod_{j=1}^{n}\left(1-\mu_{i j}\right)^{\sum_{j=1}^{R_{j}} R_{j}}\right)^{\frac{T_{i}}{\sum_{i=1}^{1} T_{i}}},\right. \\
\left.\prod_{i=1}^{1}\left(\prod_{j=1}^{n} \nu_{i j}^{\frac{R_{j}}{\sum_{j=1}^{n} R_{j}}}\right)^{\frac{T_{i}}{\sum_{i=1}^{1} T_{i}}}\right\rangle .
\end{gathered}
$$

Thus, Eq. (4) holds for $n=1 ; m=1$. Assume that Eq. (4) holds for $m=k_{1}+1, n=k_{2}$, and $m=k_{1}$, $n=k_{2}+1$, i.e.:

$$
\begin{aligned}
\bigoplus_{i=1}^{k_{1}+1} & \frac{T_{i}}{\sum_{i=1}^{m} T_{i}}\left(\bigoplus_{j=1}^{k_{2}} \frac{R_{j}}{\sum_{j=1}^{n} R_{j}} F_{e_{i j}}\right) \\
= & \left\langle 1-\prod_{i=1}^{k_{1}+1}\left(\prod_{j=1}^{k_{2}}\left(1-\mu_{i j}\right)^{\frac{R_{j}}{\sum_{j=1}^{n} R_{j}}}\right)^{\frac{T_{i}}{\sum_{i=1}^{T_{i}}}},\right. \\
& \left.\prod_{i=1}^{k_{1}+1}\left(\prod_{j=1}^{k_{2}} \nu_{i j}^{\frac{R_{j}}{\sum_{j}^{n}=1} R_{j}}\right)^{\frac{T_{i}}{\sum_{i=1}^{n} T_{i}}}\right\rangle,
\end{aligned}
$$

and:

$$
\begin{aligned}
\bigoplus_{i=1}^{k_{1}} \frac{T_{i}}{\sum_{i=1}^{m} T_{i}}\left(\bigoplus_{j=1}^{k_{2}+1} \frac{R_{j}}{\sum_{j=1}^{n} R_{j}} F_{e_{i j}}\right) \\
=\left\langle 1-\prod_{i=1}^{k_{1}}\left(\prod_{j=1}^{k_{2}+1}\left(1-\mu_{i j}\right)^{\frac{R_{j}}{\sum_{j=1}^{n} R_{j}}}\right)^{\frac{T_{i}}{\sum_{i=1}^{n_{i} T_{i}}}},\right. \\
\left.\prod_{i=1}^{k_{1}}\left(\prod_{j=1}^{k_{2}+1} \nu_{i j}^{\frac{R_{j}}{\sum_{j}^{n} R_{j}}}\right)^{\frac{T_{i}}{\sum_{i=1}^{n_{i}} T_{i}}}\right\rangle .
\end{aligned}
$$

Now:

$$
\begin{aligned}
\bigoplus_{i=1}^{k_{1}+1} & \frac{T_{i}}{\sum_{i=1}^{k_{1}+1} T_{i}}\left(\bigoplus_{j=1}^{k_{2}+1} \frac{R_{j}}{\sum_{j=1}^{k_{2}+1} R_{j}} F_{e_{i j}}\right) \\
= & \bigoplus_{i=1}^{k_{1}+1} \frac{T_{i}}{\sum_{i=1}^{k_{1}+1} T_{i}}\left(\bigoplus_{j=1}^{k_{2}} \frac{R_{j}}{\sum_{j=1}^{k_{2}+1} R_{j}} F_{e_{i j}}\right. \\
& \left.\oplus \frac{R_{k_{2}+1}}{\sum_{j=1}^{k_{2}+1} R_{j}} F_{e_{\left(k_{2}+1\right) j}}\right) \\
= & \left(\bigoplus_{i=1}^{k_{1}+1} \frac{T_{i}}{\sum_{i=1}^{k_{1}+1} T_{i}} \bigoplus_{j=1}^{k_{2}}\left(\frac{R_{j}}{\sum_{j=1}^{k_{2}+1} R_{j}} F_{e_{i j}}\right)\right)
\end{aligned}
$$

$$
\begin{aligned}
& \bigoplus_{i=1}^{k_{1}+1} \frac{T_{i}}{\sum_{i=1}^{k_{1}+1} T_{i}}\left(\frac{R_{k_{2}+1}}{\sum_{j=1}^{k_{2}+1} R_{j}} F_{e_{\left(k_{2}+1\right) j}}\right) \\
& =\left\langle 1-\prod_{i=1}^{k_{1}+1}\left(\prod_{j=1}^{k_{2}}\left(1-\mu_{i j}\right)^{\frac{R_{j}}{\sum_{j=1}^{k_{2}+1} R_{j}}}\right)^{\frac{T_{i}}{\sum_{i=1}^{k_{1}+1} T_{i}}}\right. \\
& \oplus 1-\prod_{i=1}^{k_{1}+1}\left(\left(1-\mu_{\left(k_{2}+1\right) j}\right)^{\frac{R_{k_{2}+1}}{\sum_{j=1}^{k_{2}+1} R_{j}}}\right)^{\frac{T_{i}}{\sum_{i=1}^{k_{1}+1} T_{i}}}, \\
& \prod_{i=1}^{k_{1}+1}\left(\prod_{j=1}^{k_{2}} \nu_{i j}^{\frac{R_{k_{2}+1}}{\sum_{j=1}^{k_{2}+1} R_{j}}}\right)^{\frac{T_{i}}{\sum_{i=1}^{k_{1}+1} T_{i}}} \\
& \left.\oplus \prod_{i=1}^{k_{1}+1}\left(\nu_{\left(k_{2}+1\right) j}^{\frac{R_{j}}{\sum_{j=1}^{k_{2}+1} R_{j}}}\right)^{\frac{T_{i}}{\sum_{i=1}^{k_{i}+1} T_{i}}}\right\rangle \\
& =\left\langle 1-\prod_{i=1}^{k_{1}+1}\left(\prod_{j=1}^{k_{2}+1}\left(1-\mu_{i j}\right)^{\frac{R_{j}}{\sum_{j=1}^{k_{2}+1} R_{j}}}\right)^{\frac{T_{i}}{\sum_{i=1}^{k_{1}+1} T_{i}}},\right. \\
& \left.\prod_{i=1}^{k_{1}+1}\left(\prod_{j=1}^{k_{2}+1} \nu_{i j}^{\frac{R_{j}}{\sum_{j=1}^{k_{j}+1} R_{j}}}\right)^{\frac{T_{i}}{\sum_{i=1}^{k_{i}+1} T_{i}}}\right\rangle .
\end{aligned}
$$

Therefore, Eq. (4) holds for $m=k_{1}+1$ and $n=k_{2}+$ 1 , and hence the result holds for all positive integers $m, n \geq 1$ by mathematical induction.

Example 4.1. Let $E=\left\{e_{1}, e_{2}, e_{3}\right\}$ be a set of parameters and $X=\left\{x_{1}, x_{2}, x_{3}, x_{4}\right\}$ be a set of experts giving their preferences to describe the "attractiveness of a house" in terms of IFSNs; they are all summarized as follows:

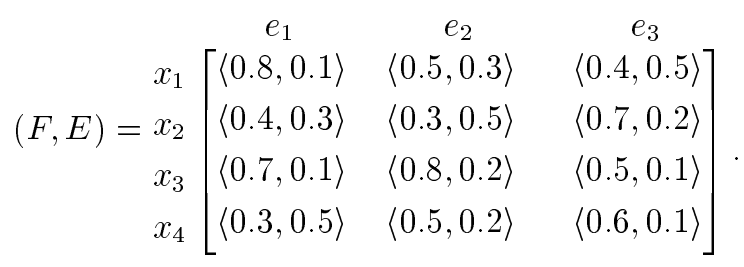

By utilizing these pieces of information and $R_{1}=$ $1, R_{j}=\prod_{l=1}^{j-1} S\left(F_{e_{i l}}\right) \quad(j=2,3), T_{1}=1, T_{i}=$ $\prod_{k=1}^{i-1} S\left(F_{e_{k}}\right)(i=2,3,4)$, we get:

$$
R=\left[\begin{array}{ccc}
1 & 0.85 & 0.60 \\
1 & 0.55 & 0.40 \\
1 & 0.80 & 0.80 \\
1 & 0.40 & 0.65
\end{array}\right], \quad T=\left[\begin{array}{c}
1 \\
0.71 \\
0.57 \\
0.78
\end{array}\right] .
$$

Thus, the aggregated IFSN by using Eq. (4) becomes: 


$$
\begin{aligned}
& \operatorname{IFSPWA}\left(F_{e_{11}}, F_{e_{12}}, \cdots, F_{e_{43}}\right) \\
&=\left\langle 1-\prod_{i=1}^{4}\left(\prod_{j=1}^{3}\left(1-\mu_{i j}\right)^{\frac{R_{j}}{\sum_{j=1}^{3} R_{j}}}\right)^{\frac{T_{i=1}^{T_{i}}}{T_{i}}},\right. \\
&\left.\prod_{i=1}^{4}\left(\prod_{j=1}^{3} \nu_{i j}^{\frac{R_{j}}{\sum_{j=1}^{3} R_{j}}}\right)^{\frac{T_{i}}{\sum_{i=1}^{4} T_{i}}}\right\rangle \\
&=\left\langle 1-\left(\left((1-0.8)^{0.41} \times(1-0.5)^{0.35} \times(1-0.4)^{0.24}\right)^{0.33}\right.\right. \\
& \times\left((1-0.4)^{0.51} \times(1-0.3)^{0.28} \times(1-0.7)^{0.20}\right)^{0.23} \\
& \times\left((1-0.7)^{0.38} \times(1-0.8)^{0.31} \times(1-0.5)^{0.31}\right)^{0.19} \\
&\left.\times\left((1-0.3)^{0.49} \times(1-0.5)^{0.20} \times(1-0.6)^{0.32}\right)^{0.25}\right) \\
&\left.\left.\times\left((0.5)^{0.49} \times(0.2)^{0.20} \times(0.1)^{0.32}\right)^{0.25}\right\rangle .5709,0.2219\right\rangle . \\
& \times\left((0.1)^{0.38} \times(0.2)^{0.31} \times(0.1)^{0.31}\right)^{0.19} \\
&\left.\left((0.1)^{0.41} \times(0.3)^{0.35} \times(0.5)^{0.24}\right)^{0.33} \times(0.5)^{0.28} \times(0.2)^{0.20}\right)^{0.23} \\
& \times(11
\end{aligned}
$$

Based on Theorem 4.1, we have the following properties for IFSNs $F_{e_{i j}}=\left\langle\mu_{i j}, \nu_{i j}\right\rangle$.

Property 4.1. (Idempotency) If $F_{e_{i j}}=F_{e}=\langle\mu, \nu\rangle$, (say) for all $i, j$ then:

$$
\operatorname{IFSPWA}\left(F_{e_{11}}, F_{e_{12}}, \ldots, F_{e_{m n}}\right)=F_{e} .
$$

Proof. Since for all $i, j, F_{e_{i j}}$ is equal, i.e. $F_{e_{i j}}=F_{e}$, then we have:

$$
\begin{aligned}
& \operatorname{IFSPWA}\left(F_{e_{11}}, F_{e_{12}}, \cdots, F_{e_{m n}}\right) \\
&=\left\langle 1-\prod_{i=1}^{m}\left(\prod_{j=1}^{n}(1-\mu)^{\frac{R_{j}}{\sum_{j=1}^{n} R_{j}}}\right)^{\frac{T_{i}}{\sum_{i=1}^{m} T_{i}}}\right. \\
&\left.\prod_{i=1}^{m}\left(\prod_{j=1}^{n} \nu^{\frac{R_{j}}{\sum_{j=1}^{n} R_{j}}}\right)^{\frac{T_{i}}{\sum_{i=1}^{m} T_{i}}}\right\rangle \\
&=\left\langle 1-\left((1-\mu)^{\sum_{j=1}^{\sum_{j=1}^{n} R_{j}}}\right)^{\frac{\sum_{i=1}^{m} T_{i}}{\sum_{i=1}^{n} T_{i}}}\right.
\end{aligned}
$$

$$
\begin{gathered}
\left.\left(\nu^{\sum_{j=1}^{n} R_{j}}\right)^{\frac{\sum_{i=1}^{m} T_{i}}{\sum_{i=1}^{n=1} T_{i}}}\right\rangle \\
=\langle 1-(1-\mu), \nu\rangle=\langle\mu, \nu\rangle=F_{e} .
\end{gathered}
$$

Property 4.2. (Boundedness) Let $F_{e_{i j}}^{-}=\left\langle\min _{i} \min _{j}\right.$ $\left.\left\{\mu_{i j}\right\}, \max _{i} \max _{j}\left\{\nu_{i j}\right\}\right\rangle$ and $F_{e_{i j}}^{+}=\left\langle\max _{i} \max _{j}\left\{\mu_{i j}\right\}, \min _{i}\right.$ $\left.\min _{j}\left\{\nu_{i j}\right\}\right\rangle$, then:

$$
F_{e_{i j}}^{-} \leq \operatorname{IFSPWA}\left(F_{e_{11}}, F_{e_{12}}, \ldots, F_{e_{m n}}\right) \leq F_{e_{i j}}^{+} .
$$

Proof. As $F_{e_{i j}}$ represents an IFSNs, so for all $i, j$ $\min _{i} \min _{j}\left\{\mu_{i j}\right\} \leq \mu_{i j} \leq \max _{i} \max _{j}\left\{\mu_{i j}\right\}$, this implies:

$$
\begin{aligned}
& 1-\max _{i} \max _{j}\left\{\mu_{i j}\right\} \leq 1-\mu_{i j} \leq 1-\min _{i} \min _{j}\left\{\mu_{i j}\right\} \\
& \Leftrightarrow\left(1-\max _{i} \max _{j}\left\{\mu_{i j}\right\}\right)^{\frac{R_{j}}{\sum_{j=1}^{n} R_{j}}} \leq\left(1-\mu_{i j}\right)^{\frac{R_{j}}{\sum_{j=1}^{n} R_{j}}} \\
& \leq\left(1-\min _{i} \min _{j}\left\{\mu_{i j}\right\}\right)^{\frac{R_{j}}{\sum_{j=1}^{n} R_{j}}} \\
& \Leftrightarrow 1-\max _{i} \max _{j}\left\{\mu_{i j}\right\} \\
& \leq \prod_{j=1}^{n}\left(1-\mu_{i j}\right)^{\frac{R_{j}}{\sum_{j=1}^{n} R_{j}}} \leq 1-\max _{i} \min _{j}\left\{\mu_{i j}\right\} \\
& \Leftrightarrow 1-\max _{i} \max _{j}\left\{\mu_{i j}\right\} \\
& \leq \prod_{i=1}^{m}\left(\prod_{j=1}^{n}\left(1-\mu_{i j}\right)^{\frac{R_{j}}{\sum_{j=1}^{n} R_{j}}}\right)^{\frac{T_{i}}{\sum_{i=1}^{m} T_{i}}} \\
& \leq 1-\min _{i} \min _{j}\left\{\mu_{i j}\right\}
\end{aligned}
$$

and hence:

$$
\begin{aligned}
\min _{i} \min _{j}\left\{\mu_{i j}\right\} & \leq 1-\prod_{i=1}^{m}\left(\prod_{j=1}^{n}\left(1-\mu_{i j}\right)^{\frac{R_{j}}{\sum_{j=1}^{n} R_{j}}}\right)^{\frac{T_{i}}{\sum_{i=1}^{m} T_{i}}} \\
& \leq \max _{i} \max _{j}\left\{\mu_{i j}\right\}
\end{aligned}
$$

Furthermore:

$$
\begin{gathered}
\min _{i} \min _{j}\left\{\nu_{i j}\right\} \leq \nu_{i j} \leq \max _{i} \max _{j}\left\{\nu_{i j}\right\} \\
\Leftrightarrow\left(\min _{i} \min _{j}\left\{\nu_{i j}\right\}\right)^{\frac{R_{j}}{\sum_{j=1}^{n} R_{j}}} \leq \nu_{i j}^{\frac{R_{j}}{\sum_{j=1}^{n} R_{j}}} \\
\leq\left(\max _{i} \max _{j}\left\{\nu_{i j}\right\}\right)^{\frac{R_{j}}{\sum_{j=1}^{n} R_{j}}}
\end{gathered}
$$




$$
\begin{aligned}
& \Leftrightarrow \min _{i} \min _{j}\left\{\nu_{i j}\right\} \leq \prod_{j=1}^{n} \nu_{i j}^{\sum_{j=1}^{R_{j}}} \leq \max _{i} \max _{j}\left\{\nu_{i j}\right\} \\
& \Leftrightarrow\left(\min _{i} \min _{j}\left\{\nu_{i j}\right\}\right)^{\frac{T_{i}}{\sum_{i=1}^{n} T_{i}}} \leq\left(\prod_{j=1}^{n} \nu_{i j}^{\frac{R_{j}}{\sum_{j=1}^{n} R_{j}}}\right)^{\frac{T_{i}}{\sum_{i=1}^{m} T_{i}}} \\
& \leq\left(\max _{i} \max _{j}\left\{\nu_{i j}\right\}\right)^{\frac{T_{i}}{\sum_{i=1}^{m} T_{i}}} \\
& \Leftrightarrow\left(\min _{i} \min _{j}\left\{\nu_{i j}\right\}\right)^{\sum_{i=1}^{\sum_{i=1}^{m} T_{i}}} \\
& \leq \prod_{i=1}^{m}\left(\prod_{j=1}^{n} \nu_{i j}^{\frac{R_{j}}{\sum_{j=1}^{n} R_{j}}}\right)^{\frac{T_{i}}{\sum_{i=1}^{m} T_{i}}} \\
& \leq\left(\max _{i} \max _{j}\left\{\nu_{i j}\right\}\right)^{\frac{\sum_{i=1}^{m} T_{i}}{\sum_{i=1} T_{i}}},
\end{aligned}
$$

and hence:

$$
\begin{aligned}
\min _{i} \min _{j}\left\{\nu_{i j}\right\} & \leq \prod_{i=1}^{m}\left(\prod_{j=1}^{n} \nu_{i j}^{\sum_{j=1}^{n} R_{j}}\right)^{\frac{T_{i}}{\sum_{i=1}^{n} T_{i}}} \\
& \leq \max _{i} \max _{j}\left\{\nu_{i j}\right\} .
\end{aligned}
$$

Let $\alpha \equiv \operatorname{IFSPWA}\left(F_{e_{11}}, F_{e_{12}}, \cdots, F_{e_{m n}}\right)=\left\langle\mu_{\alpha}, \nu_{\alpha}\right\rangle$, then we have, from Eqs. (5) and (6), $\min _{i} \min _{j}\left\{\mu_{i j}\right\} \leq$ $\mu_{\alpha} \leq \max _{i} \max _{j}\left\{\mu_{i j}\right\}$ and $\min _{i} \min _{j}\left\{\nu_{i j}\right\} \leq \nu_{\alpha} \leq$ $\max _{i} \max _{j}\left\{\nu_{i j}\right\}$. Now:

$$
\begin{aligned}
S(\alpha) & =\frac{1+\mu_{\alpha}-\nu_{\alpha}}{2} \\
& \leq \frac{1+\max _{i} \max _{j}\left\{\mu_{i j}\right\}-\min _{i} \min _{j}\left\{\nu_{i j}\right\}}{2}=S\left(F_{e_{i j}}^{+}\right), \\
S(\alpha) & =\frac{1+\mu_{\alpha}-\nu_{\alpha}}{2} \\
& \geq \frac{1+\min _{j} \min _{i}\left\{\mu_{i j}\right\}-\max _{j} \max _{i}\left\{\nu_{i j}\right\}}{2}=S\left(F_{e_{i j}}^{-}\right) .
\end{aligned}
$$

In that direction, three cases are considered:

- Case 1: If $S\left(F_{e_{i j}}\right)<S\left(F_{e_{i j}}^{+}\right)$and $S\left(F_{e_{i j}}\right)>S\left(F_{e_{i j}}^{-}\right)$, then by comparison laws between two IFSNs, we have:

$$
F_{e_{i j}}^{-} \leq \operatorname{IFSPWA}\left(F_{e_{11}}, F_{e_{12}}, \cdots, F_{e_{n m}}\right) \leq F_{e_{i j}}^{+} .
$$

- Case 2: If $S\left(F_{e_{i j}}\right)=S\left(F_{e_{i j}}^{+}\right)$, i.e., $\mu_{\alpha}-\nu_{\alpha}=$ $\max _{j} \max _{i}\left\{\mu_{i j}\right\}-\min _{j} \min _{i}\left\{\nu_{i j}\right\}$, then by the above inequalities, we have $\mu_{\alpha}=\max _{j} \max _{i}\left\{\mu_{i j}\right\}$ and $\nu_{\alpha}=$ $\min _{j} \min _{i}\left\{\nu_{i j}\right\}$. Thus:

$$
\begin{aligned}
H(\alpha) & =\mu_{\alpha}+\nu_{\alpha}=\max _{j} \max _{i}\left\{\mu_{i j}\right\}+\min _{j} \min _{i}\left\{\nu_{i j}\right\} \\
& =H\left(F_{e_{i j}}^{+}\right)
\end{aligned}
$$

then by comparison laws between two IFSNs, we have:

$$
\operatorname{IFSPWA}\left(F_{e_{11}}, F_{e_{12}}, \cdots, F_{e_{n m}}\right)=F_{e_{i j}}^{+} .
$$

Case 3: If $S\left(F_{e_{i j}}\right)=S\left(F_{e_{i j}}^{-}\right)$, i.e. $\mu_{\alpha}-\nu_{\alpha}=$ $\min _{j} \min _{i}\left\{\mu_{i j}\right\}-\max _{j} \max _{i}\left\{\nu_{i j}\right\}$, then by the above inequalities, we have $\mu_{\alpha}=\min _{j} \min _{i}\left\{\mu_{i j}\right\}$ and $\nu_{\alpha}=$ $\max _{j} \max _{i}\left\{\nu_{i j}\right\}$. Thus:

$$
\begin{aligned}
H(\alpha) & =\mu_{\alpha}+\nu_{\alpha}=\min _{j} \min _{i}\left\{\mu_{i j}\right\}+\max _{j} \max _{i}\left\{\nu_{i j}\right\} \\
& =H\left(F_{e_{i j}}^{-}\right),
\end{aligned}
$$

Then, it follows that:

$$
\operatorname{IFSPWA}\left(F_{e_{11}}, F_{e_{12}}, \cdots, F_{e_{n m}}\right)=F_{e_{i j}}^{-} .
$$

Hence, property holds.

Property 4.3. (Monotonicity) Let $F_{e_{i j}}^{\prime}$ be another IFSNs, such that $F_{e_{i j}} \leq F_{e_{i j}}^{\prime}$ for all $i, j$, then:

$$
\begin{aligned}
\operatorname{IFSPWA} & \left(F_{e_{11}}, F_{e_{12}}, \cdots, F_{e_{m n}}\right) \\
& \leq \operatorname{IFSPWA}\left(F_{e_{11}}^{\prime}, F_{e_{12}}^{\prime}, \cdots, F_{e_{m n}}^{\prime}\right)
\end{aligned}
$$

Proof. Proof is as similar as that of Property 4.2, so we omit it here.

\subsection{Intuitionistic Fuzzy Soft Prioritized Ordered Weighted Average (IFSPOWA) operator}

In this section, we will introduce an ordered weighted averaging PA operator named as IFSPOWA operator for the collections of IFSNs.

Definition 4.2. Let $F_{e_{i j}}=\left\langle\mu_{i j}, \nu_{i j}\right\rangle(i=1,2, \ldots, m$; $j=1,2, \ldots, n)$ be IFSNs. Then, an Intuitionistic Fuzzy Soft Prioritized Ordered Weighted Average (IFSPOWA) operator is defined as follows:

$$
\begin{aligned}
\operatorname{IFSPOWA}\left(F_{e_{11}}, F_{e_{12}}, \cdots, F_{e_{m n}}\right) \\
=\bigoplus_{i=1}^{m} \frac{T_{i}}{\sum_{i=1}^{m} T_{i}}\left(\bigoplus_{j=1}^{n} \frac{R_{j}}{\sum_{j=1}^{n} R_{j}} F_{e_{\delta(i) \gamma(j)}}\right),
\end{aligned}
$$


where $R_{j}=\prod_{l=1}^{j-1} S\left(F_{e_{i \gamma(l)}}\right)$ and $T_{i}=\prod_{k=1}^{i-1} S\left(F_{\left.e_{\delta(k)}\right)}\right)$. Let $R_{1}=1, T_{1}=1$, and $S\left(F_{e}\right)$ represents score function of IFSN $F_{e}$. Also, $\delta$ and $\gamma$ are permutations of $(1,2, \cdots, m)$ and $(1,2, \ldots, n)$, such that $e_{\delta(i) j} \geq$ $e_{\delta(i-1) j}$ and $e_{i \gamma(j)} \geq e_{i \gamma(j-1)}$ for $i=2,3, \ldots, m ; j=$ $2,3, \ldots, n$.

Theorem 4.2. The aggregated value of all IFSNs $F_{e_{i j}}=\left\langle\mu_{i j}, \nu_{i j}\right\rangle(i=1,2, \ldots, m ; j=1,2, \ldots, n)$ by using IFSPOWA operator is still an IFSN and is defined as follows:

$$
\begin{aligned}
& \operatorname{IFSPOWA}\left(F_{e_{11}}, F_{e_{12}}, \ldots, F_{e_{m n}}\right) \\
& =\left\langle 1-\prod_{i=1}^{m}\left(\prod_{j=1}^{n}\left(1-\mu_{\delta(i) \gamma(j)}\right)^{\frac{R_{j}}{\sum_{j=1}^{n} R_{j}}}\right)^{\frac{T_{i}}{\sum_{i=1}^{m} T_{i}}},\right. \\
& \left.\prod_{i=1}^{m}\left(\prod_{j=1}^{n} \nu_{\delta(i) \gamma(j)}^{\frac{R_{j}}{\sum_{j=1}^{n} R_{j}}}\right)^{\frac{T_{i}}{\sum_{i=1}^{m} T_{i}}}\right\rangle,
\end{aligned}
$$

where $R_{1}=1, T_{1}=1, R_{j}=\prod_{l=1}^{j-1} S\left(F_{e_{i \gamma(l)}}\right)(j=$ $2,3, \cdots, n)$, and $T_{i}=\prod_{k=1}^{i-1} S\left(F_{e_{\delta(k)}}\right)(i=2,3, \cdots, m)$, where $S\left(F_{e}\right)$ represents score function of IFSN $F_{e}$.

Proof. Proof of this theorem is the same as that of Theorem 4.1.

Example 4.2. As given in Example 4.1, $(F, E)$ is an IFSN. Now, by using Eq. (1), we have $S\left(e_{11}\right)=$ $0.85, S\left(e_{12}\right)=0.60$ and $S\left(e_{13}\right)=0.45$. Thus, $S\left(e_{11}\right)>S\left(e_{12}\right)>S\left(e_{13}\right)$, and therefore, $F_{e_{1 \gamma(1)}}=$ $\langle 0.8,0.1\rangle, F_{e_{1 \gamma(2)}}=\langle 0.5,0.3\rangle$ and $F_{e_{1 \gamma(3)}}=\langle 0.4,0.5\rangle$. Similarly, we can find the other $F_{e_{i \gamma}(j)}$. Hence, the ordered matrix of $e_{i \gamma(j)}$ 's is given as follows:

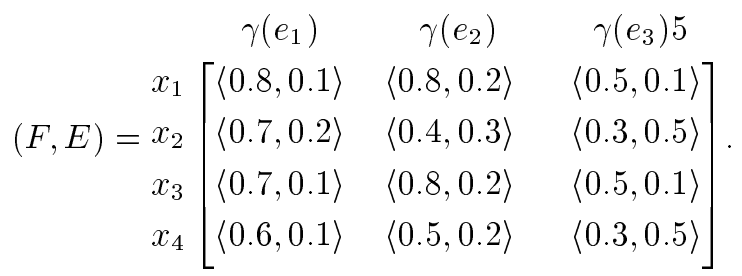

Furthermore:

$$
\begin{aligned}
& S\left(F_{e_{1 \gamma(1)}}\right)=0.85, \quad S\left(F_{e_{2 \gamma(1)}}\right)=0.75, \\
& S\left(F_{e_{3 \gamma(1)}}\right)=0.8, \quad S\left(F_{e_{4 \gamma(1)}}\right)=0.75,
\end{aligned}
$$

therefore:

$$
S\left(F_{e_{1 \gamma(1)}}\right)>S\left(F_{e_{3 \gamma(1)}}\right)>S\left(F_{e_{2 \gamma(1)}}\right)>S\left(F_{e_{4 \gamma(1)}}\right) .
$$

Thus:

$$
\begin{array}{ll}
F_{e_{\delta(1) \gamma(1)}}=\langle 0.8,0.1\rangle, & F_{e_{\delta(2) \gamma(1)}}=\langle 0.7,0.1\rangle, \\
F_{e_{\delta(3) \gamma(1)}}=\langle 0.7,0.2\rangle, & F_{e_{\delta(4) \gamma(1)}}=\langle 0.6,0.1\rangle .
\end{array}
$$

Then, the ordered matrix for $\operatorname{IFSS}(F, E)$ is:

$$
\begin{aligned}
& \gamma\left(e_{1}\right) \quad \gamma\left(e_{2}\right) \quad \gamma\left(e_{3}\right) \\
& \begin{aligned}
& \delta\left(x_{1}\right) \\
&(F, E)= \delta\left(x_{2}\right)
\end{aligned}\left[\begin{array}{ccc}
\langle 0.8,0.1\rangle & \langle 0.8,0.2\rangle & \langle 0.5,0.1\rangle \\
\delta\left(x_{3}\right) & \delta\left(x_{4}\right)
\end{array}\left[\begin{array}{lll}
\langle 0.7,0.1\rangle & \langle 0.5,0.2\rangle & \langle 0.4,0.5\rangle \\
\langle 0.6,0.1\rangle & \langle 0.5,0.3\rangle & \langle 0.3,0.5\rangle \\
& \langle 0.4\rangle & \langle 0.3,0.5\rangle
\end{array}\right] .\right.
\end{aligned}
$$

Now, by using $R_{1}=1, T_{1}=1, R_{j}=\prod_{l=1}^{j-1} S\left(F_{e_{i \gamma(l)}}\right)$ $(j=2,3)$, and $T_{i}=\prod_{k=1}^{i-1} S\left(F_{e_{\delta(k)}}\right)(i=2,3,4)$, we get:

$$
R=\left[\begin{array}{lll}
1 & 0.85 & 0.80 \\
1 & 0.80 & 0.65 \\
1 & 0.75 & 0.60 \\
1 & 0.75 & 0.55
\end{array}\right], \quad T=\left[\begin{array}{c}
1 \\
0.7868 \\
0.6376 \\
0.6583
\end{array}\right]
$$

Hence:

$$
\begin{aligned}
& \operatorname{IFSpOWA}\left(F_{e_{11}}, F_{e_{12}}, \cdots, F_{e_{43}}\right) \\
& =\left\langle 1-\prod_{i=1}^{4}\left(\prod_{j=1}^{3}\left(1-\mu_{\delta(i) \gamma(j)}\right)^{\frac{R_{j}}{\sum_{j=1}^{3} R_{j}}}\right)^{\frac{T_{i}}{\sum_{i=1}^{4} T_{i}}}\right. \\
& \left.\quad \prod_{i=1}^{4}\left(\prod_{j=1}^{3} \nu_{\delta(i) \gamma(j)}^{\frac{R_{j}}{\sum_{j=1}^{3} R_{j}}}\right)^{\frac{T_{i}}{\sum_{i=1}^{4} T_{i}}}\right\rangle \\
& =\langle 0.5964,0.2127\rangle .
\end{aligned}
$$

As similar to IFSPWA operator, IFSPOWA operator also satisfies some properties.

Property 4.4. Let $F_{e_{i j}}$ and $F_{e_{i j}}^{\prime},(i=1,2, \cdots, m$; $j=1,2, \cdots, n)$ be two collections of IFSNs, then:

(i) (Idempotancy) If all $F_{e_{i j}}=F_{e}$, then IFSPOWA $\left(F_{e_{11}}, F_{e_{12}}, \cdots, F_{e_{m n}}\right)$

(ii) (Boundedness) Let $F_{e_{i j}}^{-}=\left\langle\min _{i} \min _{j}\left\{\mu_{i j}\right\}, \max _{i}\right.$ $\left.\max _{j}\left\{\nu_{i j}\right\}\right\rangle$ and $F_{e_{i j}}^{+}=\left\langle\max _{i} \max _{j}\left\{\mu_{i j}\right\}, \min _{i} \min _{j}\right.$ $\left.\left\{\nu_{i j}\right\}\right\rangle$ then $F_{e_{i j}}^{-} \leq \operatorname{IFSPOWA}\left(F_{e_{11}}, F_{e_{12}}, \cdots\right.$, $\left.F_{e_{m n}}\right) \leq F_{e_{i j}}^{+}$

(iii) (Monotonicity) If $F_{e_{i j}} \leq F_{e_{i j}}^{\prime}$, for all $i, j$, then $\operatorname{IFSPOWA}\left(F_{e_{11}}, F_{e_{12}}, \cdots, F_{e_{m n}}\right) \leq$ $\operatorname{IFSPOWA}\left(F_{e_{11}}^{\prime}, F_{e_{12}}^{\prime}, \cdots, F_{e_{m n}}^{\prime}\right)$. 


\subsection{Intuitionistic Fuzzy Soft Prioritized Weighted Geometric (IFSPWG) operator}

In this section, we will introduce the weighted geometric $\mathrm{PA}$ operator named as IFSPWG operator for the collections of IFSNs.

Definition 4.3. Let $F_{e_{i j}}=\left\langle\mu_{i j}, \nu_{i j}\right\rangle,(i=1,2, \cdots$, $m ; j=1,2, \cdots, n)$ be collections of IFSNs. Then, an IFSPWG operator is defined as follows:

$$
\begin{aligned}
\operatorname{IFSPWG} & \left(F_{e_{11}}, F_{e_{12}}, \cdots, F_{e_{m n}}\right) \\
= & \bigotimes_{i=1}^{m}\left(\bigotimes_{j=1}^{n} F_{e_{i j}}^{\frac{R_{j}}{\sum_{j=1}^{n} R_{j}}}\right)^{\frac{T_{i}}{\sum_{i=1}^{m_{i}} T_{i}}},
\end{aligned}
$$

where $R_{1}=1, T_{1}=1, R_{j}=\prod_{l=1}^{j-1} S\left(F_{e_{i l}}\right)(j=$ $2,3, \cdots, n)$, and $T_{i}=\prod_{k=1}^{i-1} S\left(F_{e_{k}}\right)(i=2,3, \cdots, m)$; $S\left(F_{e}\right)$ represents the score function of IFSN $F_{e}$.

Theorem 4.3. The aggregated value of all IFSNs $F_{e_{i j}}$ by using IFSPWG operator is still an IFSN defined as follows:

$$
\begin{aligned}
& \operatorname{IFSPWG}\left(F_{e_{11}}, F_{e_{12}}, \cdots, F_{e_{m n}}\right) \\
& =\left\langle\prod_{i=1}^{m}\left(\prod_{j=1}^{n} \frac{R_{j}}{\sum_{i j}^{n} R_{j}}\right)^{\frac{T_{i}}{\sum_{i=1}^{m} T_{i}}},\right. \\
& \left.\quad 1-\prod_{i=1}^{m}\left(\prod_{j=1}^{n}\left(1-\nu_{i j}\right)^{\frac{R_{j}}{\sum_{j=1}^{n} R_{j}}}\right)^{\frac{T_{i}}{\sum_{i=1}^{n} T_{i}}}\right\rangle .
\end{aligned}
$$

Proof. We prove this result by mathematical induction on $n$ and $m$. For $n=1$ :

$$
\begin{gathered}
\operatorname{IFSPWG}\left(F_{e_{11}}, F_{e_{21}}, \cdots, F_{e_{m 1}}\right)=\bigotimes_{i=1}^{m} F_{e_{i 1}}^{\frac{T_{i}}{\sum_{i=1}^{m} T_{i}}} \\
=\left\langle\prod_{i=1}^{m} \mu_{i 1}^{\frac{T_{i}}{\sum_{i=1}^{m_{i} T_{i}}}}, 1-\prod_{i=1}^{m}\left(1-\nu_{i 1}\right)^{\frac{T_{i}}{\sum_{i=1}^{m_{1}} T_{i}}}\right\rangle \\
=\left\langle\prod_{i=1}^{m}\left(\prod_{j=1}^{1} \mu_{i j}^{\frac{R_{j}}{\sum_{j=1}^{1} R_{j}}}\right)^{\frac{T_{i}}{\sum_{i=1}^{m} T_{i}}},\right. \\
\left.1-\prod_{i=1}^{m}\left(\prod_{j=1}^{1}\left(1-\nu_{i j}\right)^{\frac{R_{j}}{\sum_{j=1}^{1} R_{j}}}\right)^{\frac{T_{i}}{\sum_{i=1}^{m} T_{i}}}\right\rangle
\end{gathered}
$$

and for $m=1$ :

$$
\operatorname{IFSPWG}\left(F_{e_{11}}, F_{e_{12}}, \cdots, F_{e_{1 n}}\right)=\bigotimes_{j=1}^{n} F_{e_{1 j}}^{\frac{R_{j}}{\sum_{j=1}^{n} R_{j}}}
$$

$$
\begin{aligned}
= & \left\langle\prod_{j=1}^{n} \mu_{1 j}^{\frac{R_{j}}{\sum_{j=1}^{n} R_{j}}}, 1-\prod_{j=1}^{n}\left(1-\nu_{1 j}\right)^{\frac{R_{j}}{\sum_{j=1}^{n} R_{j}}}\right\rangle \\
= & \left\langle\prod_{i=1}^{1}\left(\prod_{j=1}^{n} \mu_{i j}^{\frac{R_{j}}{\sum_{j=1}^{n} R_{j}}}\right)^{\frac{T_{i}}{\sum_{i=1}^{1} T_{i}}},\right. \\
& \left.1-\prod_{i=1}^{1}\left(\prod_{j=1}^{n}\left(1-\nu_{i j}\right)^{\frac{R_{j}}{\sum_{j=1}^{n} R_{j}}}\right)^{\frac{T_{i}}{\sum_{i=1}^{1} T_{i}}}\right\rangle .
\end{aligned}
$$

Assume that the result is true for $m=k_{1}+1, n=k_{2}$, and $m=k_{1}, n=k_{2}+1$ i.e.:

$$
\begin{aligned}
\bigotimes_{i=1}^{k_{1}+1} & \left(\bigotimes_{j=1}^{k_{2}} F_{e_{i j}}^{\frac{R_{j}}{\sum_{j=1}^{k_{2}} R_{j}}}\right)^{\frac{T_{i}}{\sum_{i=1}^{k_{1}+1} T_{i}}} \\
= & \left\langle\prod_{i=1}^{k_{1}+1}\left(\prod_{j=1}^{k_{2}} \mu_{i j}^{\frac{R_{j}}{\sum_{j=1}^{k_{2}} R_{j}}}\right)^{\frac{T_{i}}{\sum_{i=1}^{k_{i}+1} T_{i}}},\right. \\
& \left.1-\prod_{i=1}^{k_{1}+1}\left(\prod_{j=1}^{k_{2}}\left(1-\nu_{i j}\right)^{\frac{R_{j}}{\sum_{j=1}^{k_{2}} R_{j}}}\right)^{\frac{T_{i}}{\sum_{i=1}^{k_{1}+1} T_{i}}}\right\rangle
\end{aligned}
$$

and:

$$
\begin{aligned}
\bigotimes_{i=1} & \left(\bigotimes_{j=1}^{k_{1}} F_{e_{i j}}^{\frac{k_{j}}{\sum_{j}^{k_{2}+1} R_{j}}}\right)^{\frac{T_{i}}{\sum_{i=1}^{k_{1}} T_{i}}} \\
= & \left\langle\prod_{i=1}^{k_{1}}\left(\prod_{j=1}^{k_{2}+1} \mu_{i j}^{\frac{R_{j}}{\sum_{j=1}^{k_{2}+1} R_{j}}}\right)^{\frac{T_{i}}{\sum_{i=1}^{k_{1}} T_{i}}},\right. \\
& \left.1-\prod_{i=1}^{k_{1}}\left(\prod_{j=1}^{k_{2}+1}\left(1-\nu_{i j}\right)^{\frac{R_{j}}{\sum_{j=1}^{k_{2}+1} R_{j}}}\right)^{\frac{T_{i}}{\sum_{i=1}^{k_{1}} T_{i}}}\right\rangle .
\end{aligned}
$$

Now, to prove that the result is true for $m=k_{1}+1$ and $n=k_{2}+1$ :

$$
\begin{aligned}
& \bigotimes_{i=1}^{k_{1}+1}\left(\bigotimes_{j=1}^{k_{2}+1} \frac{R_{j}}{F_{e_{i j}}^{\sum_{j} k_{1}+1} R_{j}}\right)^{\frac{T_{i}}{\sum_{i=1}^{k_{1}+1} T_{i}}} \\
& =\bigotimes_{i=1}^{k_{1}+1}\left(\bigotimes_{j=1}^{k_{2}} F_{e_{i j}}^{\frac{R_{j}}{\sum_{j=1}^{k_{2}+1} R_{j}}} \otimes F_{e_{\left(k_{2}+1\right) j}}^{\frac{R_{k_{2}+1}}{\sum_{j=1}^{k_{2}+1} R_{j}}}\right)^{\frac{T_{i}}{\sum_{i=1}^{k_{1}+1} T_{i}}} \\
& =\left(\bigotimes_{i=1}^{k_{1}+1}\left(\bigotimes_{j=1}^{k_{2}} \frac{R_{j}}{F_{e_{i j}}^{\sum_{j=1}^{k_{2}+1} R_{j}}}\right)^{\frac{T_{i}}{\sum_{i=1}^{k_{1}+1} T_{i}}}\right)
\end{aligned}
$$




$$
\begin{aligned}
& \bigotimes_{i=1}^{k_{1}+1}\left(F_{E_{\left(k_{2}+1\right) j}}^{\frac{R_{k_{2}+1}}{\sum_{j 21}^{k_{2}+1} R_{j}}}\right)^{\frac{T_{i}}{\sum_{i=1}^{k_{1}+1} T_{i}}} \\
& =\left\langle\prod_{i=1}^{k_{1}+1}\left(\prod_{j=1}^{k_{2}} \mu_{i j}^{\frac{R_{j}}{\sum_{j=1}^{k_{2}+1} R_{j}}}\right)^{\frac{T_{i}}{\sum_{i=1}^{k_{1}+1} T_{i}}}\right. \\
& \otimes \prod_{i=1}^{k_{1}+1}\left(\mu_{\left(k_{2}+1\right) j}^{\frac{R_{k_{2}+1}}{\sum_{j=1}^{k_{2}+1} R_{j}}}\right)^{\frac{T_{i}}{\sum_{i=1}^{k_{1}+1} T_{i}}} \\
& 1-\prod_{i=1}^{k_{1}+1}\left(\prod_{j=1}^{k_{2}}\left(1-\nu_{i j}\right)^{\frac{R_{j}}{\sum_{j=1}^{k_{2}+1} R_{j}}}\right)^{\frac{T_{i}}{\sum_{i=1}^{k_{1}+1} T_{i}}} \\
& \left.\otimes 1-\prod_{i=1}^{k_{1}+1}\left(\left(1-\nu_{\left(k_{2}+1\right) j}\right)^{\frac{R_{k_{2}+1}}{\sum_{j=1}^{k_{2}+1} R_{j}}}\right)^{\frac{T_{i}}{\sum_{i=1}^{k_{1}+1} T_{i}}}\right\rangle \\
& =\left\langle\prod_{i=1}^{k_{1}+1}\left(\prod_{j=1}^{k_{2}+1} \mu_{i j}^{\frac{R_{j}}{\sum_{j=1}^{k_{2}+1} R_{j}}}\right)^{\frac{T_{i}}{\sum_{i=1}^{k_{1}+1} T_{i}}}\right. \\
& \left.1-\prod_{i=1}^{k_{1}+1}\left(\prod_{j=1}^{k_{2}+1}\left(1-\nu_{i j}\right)^{\frac{R_{j}}{\sum_{j=1}^{k_{2}+1} R_{j}}}\right)^{\frac{T_{i}}{\sum_{i=1}^{k_{1}+1} T_{i}}}\right\rangle .
\end{aligned}
$$

It shows that the result holds for $m=k_{1}+1$ and $n=k_{2}+1$; thus, by mathematical induction, the result holds for all $m, n \geq 1$.

Example 4.3. Let $X=\left\{x_{1}, x_{2}, x_{3}, x_{4}\right\}$ be a set of experts giving their preferences for describing their "usage of a mobile" on certain parameters $E=\left\{e_{1}, e_{2}, e_{3}\right\}$. Then, IFSS $(F, E)$ is defined as follows:

$$
(F, E)=x_{2}\left[\begin{array}{ccc}
e_{1} & e_{2} & e_{3} \\
& x_{3} \\
x_{4}
\end{array}\left[\begin{array}{ccc}
\langle 0.7,0.2\rangle & \langle 0.4,0.1\rangle & \langle 0.6,0.1\rangle \\
\langle 0.7,0.1\rangle & \langle 0.5,0.3\rangle & \langle 0.3,0.6\rangle \\
\langle 0.6,0.1\rangle & \langle 0.5,0.2\rangle & \langle 0.8,0.1\rangle \\
\langle 0.6,0.4\rangle & \langle 0.8,0.1\rangle & \langle 0.6,0.1\rangle
\end{array}\right] .\right.
$$

Based on these data, we get:

$$
R=\left[\begin{array}{lll}
1 & 0.75 & 0.65 \\
1 & 0.80 & 0.60 \\
1 & 0.75 & 0.65 \\
1 & 0.60 & 0.85
\end{array}\right], \quad T=\left[\begin{array}{c}
1 \\
0.7103 \\
0.5910 \\
0.7401
\end{array}\right],
$$

and hence by using Eq. (10), we get:

$$
\operatorname{IFSPWG}\left(F_{e_{11}}, F_{e_{12}}, \cdots, F_{e_{43}}\right)
$$

$$
\begin{aligned}
& =\left\langle\prod_{i=1}^{4}\left(\prod_{j=1}^{3} \mu_{i j}^{\frac{R_{j}}{\sum_{j=1}^{3} R_{j}}}\right)^{\frac{T_{i}}{\sum_{i=1}^{4} T_{i}}},\right. \\
& \left.\quad 1-\prod_{i=1}^{4}\left(\prod_{j=1}^{3}\left(1-\nu_{i j}\right)^{\frac{R_{j}}{\sum_{j=1}^{3} R_{j}}}\right)^{\frac{T_{i}}{\sum_{i=1}^{4} T_{i}}}\right\rangle \\
& =\langle 0.5771,0.2101\rangle
\end{aligned}
$$

As similar to IFSPWA operator, IFSPWG also satisfies some properties for the collection of IFSNs $F_{e_{i j}}(i=$ $1,2, \cdots, m ; j=1,2, \cdots, n)$ which are as follows:

Property 4.5. (Idempotency) If all $F_{e_{i j}}=F_{e}=$ $\langle\mu, \nu\rangle$, then $\operatorname{IFSPWG}\left(F_{e_{11}}, F_{e_{12}}, \cdots, F_{e_{m n}}\right)=F_{e}$.

Proof. Since all $F_{e_{i j}}$ are equal, i.e. $F_{e_{i j}}=F_{e}$, then we have:

$$
\begin{aligned}
& \operatorname{IFSPWG}\left(F_{e_{11}}, F_{e_{12}}, \cdots, F_{e_{m n}}\right) \\
& =\left\langle\prod_{i=1}^{m}\left(\prod_{j=1}^{n} \mu^{\frac{R_{j}}{\sum_{j=1}^{n} R_{j}}}\right)^{\frac{T_{i}}{\sum_{i=1}^{m} T_{i}}},\right. \\
& \left.\quad 1-\prod_{i=1}^{m}\left(\prod_{j=1}^{n}(1-\nu)^{\frac{R_{j}}{\sum_{j=1}^{n} R_{j}}}\right)^{\frac{T_{i}}{\sum_{i=1}^{m} T_{i}}}\right\rangle \\
& =\left\langle\left(\mu^{\sum_{j=1}^{n} R_{j}^{n} R_{j}}\right)^{\frac{\sum_{i=1}^{m} T_{i}}{\sum_{i=1}^{n} T_{i}}},\right. \\
& \left.\quad 1-\left((1-\nu)^{\sum_{j=1}^{\sum_{j=1}^{n} R_{j}}}\right)^{\sum_{i=1}^{\sum_{i=1}^{m} T_{i}}}\right\rangle \\
& =\langle\mu, 1-(1-\nu)\rangle=\langle\mu, \nu\rangle=F_{e} .
\end{aligned}
$$

Property 4.6. (Boundedness) Let $F_{e_{i j}}^{-}=\left\langle\min _{i} \min _{j}\right.$ $\left.\left\{\mu_{i j}\right\}, \max _{i} \max _{j}\left\{\nu_{i j}\right\}\right\rangle$ and $F_{e_{i j}}^{+}=\left\langle\max _{i} \max _{j}\left\{\mu_{i j}\right\}, \min _{i}\right.$ $\left.\min _{j}\left\{\nu_{i j}\right\}\right\rangle$, then:

$$
F_{e_{i j}}^{-} \leq \operatorname{IFSPWG}\left(F_{e_{11}}, F_{e_{12}}, \cdots, F_{e_{m n}}\right) \leq F_{e_{i j}}^{+}
$$

Proof. Since for all $i, j$, we have:

$$
\begin{aligned}
& \min _{i} \min _{j}\left\{\mu_{i j}\right\} \leq \mu_{i j} \leq \max _{i} \max _{j}\left\{\mu_{i j}\right\} \\
& \Leftrightarrow \min _{i} \min _{j}\left\{\mu_{i j}\right\}
\end{aligned}
$$




$$
\begin{aligned}
& \leq \prod_{j=1}^{n} \mu_{i j}^{\frac{R_{j}}{\sum_{j=1}^{n} R_{j}}} \leq \max _{i} \max _{j}\left\{\mu_{i j}\right\} \\
& \Leftrightarrow\left(\min _{i} \min _{j}\left\{\mu_{i j}\right\}\right)^{\frac{T_{i}}{\sum_{i=1}^{m_{i}} T_{i}}} \\
& \leq\left(\prod_{j=1}^{n} \mu_{i j}^{\frac{R_{j}}{\sum_{j=1}^{n} R_{j}}}\right)^{\frac{T_{i}}{\sum_{i=1}^{n} T_{i}}} \\
& \leq\left(\max _{i} \max _{j}\left\{\mu_{i j}\right\}\right)^{\frac{T_{i}}{\sum_{i=1}^{n_{i}} T_{i}}},
\end{aligned}
$$

which implies that:

$$
\begin{aligned}
\min _{i} \min _{j}\left\{\mu_{i j}\right\} & \leq \prod_{i=1}^{m}\left(\prod_{j=1}^{n} \frac{R_{j}}{\mu_{i j}^{n} R_{j}^{n}}\right)^{\frac{T_{i}}{\sum_{i=1}^{n_{i} T_{i}}}} \\
& \leq \max _{i} \max _{j}\left\{\mu_{i j}\right\} .
\end{aligned}
$$

Furthermore:

$$
\begin{aligned}
& \min _{i} \min _{j}\left\{\nu_{i j}\right\} \leq \nu_{i j} \leq \max _{i} \max _{j}\left\{\nu_{i j}\right\} \\
& \Leftrightarrow 1-\max _{i} \max _{j}\left\{\nu_{i j}\right\} \leq \prod_{j=1}^{n}\left(1-\nu_{i j}\right)^{\frac{R_{j}}{\sum_{j=1}^{n} R_{j}}} \\
& \leq 1-\min _{i} \min _{j}\left\{\nu_{i j}\right\} \\
& \Leftrightarrow\left(1-\max _{i} \max _{j}\left\{\nu_{i j}\right\}\right)^{\frac{\sum_{i=1}^{m} T_{i}}{\sum_{i=1}^{i=1} T_{i}}} \\
& \leq \prod_{i=1}^{m}\left(\prod_{j=1}^{n}\left(1-\nu_{i j}\right)^{\frac{R_{j}}{\sum_{j=1}^{n} R_{j}}}\right)^{\frac{T_{i}}{\sum_{i=1}^{m} T_{i}}} \\
& \leq\left(1-\min _{i} \min _{j}\left\{\nu_{i j}\right\}\right)^{\frac{\sum_{i=1}^{m} T_{i}}{\sum_{i=1}^{i n} T_{i}}} \\
& \Leftrightarrow 1-\max _{i} \max _{j}\left\{\nu_{i j}\right\} \\
& \leq \prod_{i=1}^{m}\left(\prod_{j=1}^{n}\left(1-\nu_{i j}\right)^{\frac{R_{j}}{\sum_{j=1}^{n} R_{j}}}\right)^{\frac{T_{i}}{\sum_{i=1}^{n} T_{i}}} \\
& \leq 1-\min _{i} \min _{j}\left\{\nu_{i j}\right\},
\end{aligned}
$$

which implies that:

$$
\begin{aligned}
\min _{i} \min _{j}\left\{\nu_{i j}\right\} & \leq 1-\prod_{i=1}^{m}\left(\prod_{j=1}^{n}\left(1-\nu_{i j}\right)^{\frac{R_{j}}{\sum_{j=1}^{n} R_{j}}}\right)^{\frac{T_{i}}{\sum_{i=1}^{m_{i}}}} \\
& \leq \max _{i} \max _{j}\left\{\nu_{i j}\right\} .
\end{aligned}
$$

Let $\alpha \equiv \operatorname{IFSPWG}\left(F_{e_{11}}, F_{e_{12}}, \cdots, F_{e_{m n}}\right)=\left\langle\mu_{\alpha}, \nu_{\alpha}\right\rangle$. Thus, from Eqs. (11) and (12), we get $\min _{i} \min _{j}\left\{\mu_{i j}\right\} \leq$ $\mu_{\alpha} \leq \max _{i} \max _{j}\left\{\mu_{i j}\right\}, \min _{i} \min _{j}\left\{\nu_{i j}\right\} \leq \nu_{\alpha} \leq \max _{i}$ $\max _{j}\left\{\nu_{i j}\right\}$. Now:

$$
\begin{aligned}
S(\alpha) & =\frac{1+\mu_{\alpha}-\nu_{\alpha}}{2} \\
& \leq \frac{1+\max _{i} \max _{j}\left\{\mu_{i j}\right\}-\min _{i} \min _{j}\left\{\nu_{i j}\right\}}{2}=S\left(F_{e_{i j}}^{+}\right), \\
S(\alpha) & =\frac{1+\mu_{\alpha}-\nu_{\alpha}}{2} \\
& \geq \frac{1+\min _{j} \min _{i}\left\{\mu_{i j}\right\}-\max _{j} \max _{i}\left\{\nu_{i j}\right\}}{2}=S\left(F_{e_{i j}}^{-}\right) .
\end{aligned}
$$

Hence, by comparison law, we get:

$$
F_{e_{i j}}^{-} \leq \operatorname{IFSPWG}\left(F_{e_{11}}, F_{e_{12}}, \cdots, F_{e_{m n}}\right) \leq F_{e_{i j}}^{+} .
$$

Property 4.7. (Monotonicity) Let $F_{e_{i j}}^{\prime}$ be another collection of IFSNs, such that $F_{e_{i j}} \leq F_{e_{i j}}^{\prime}$ for $i, j$, then $\operatorname{IFSPWG}\left(F_{e_{11}}, F_{e_{12}}, \cdots, F_{e_{m n}}\right) \leq \operatorname{IFSP}$ $\operatorname{WG}\left(F_{e_{11}}^{\prime}, F_{e_{12}}^{\prime}, \cdots, F_{e_{m n}}^{\prime}\right)$.

Proof. Proof of this property is the same as that of Property 4.6, so it is omitted here.

\subsection{Intuitionistic Fuzzy Soft Prioritized Ordered Weighted Geometric (IFSPOWG) operator}

In this section, we will introduce an ordered weighted geometric PA operator named as IFSPOWG operator for the collections of IFSNs.

Definition 4.4. Let $F_{e_{i j}}=\left\langle\mu_{i j}, \nu_{i j}\right\rangle(i=1,2, \cdots$, $m ; j=1,2, \cdots, n)$ be the collections of IFSNs. Then, an IFSPOWG operator is defined as follows:

$$
\begin{aligned}
\operatorname{IFSPOWG} & \left(F_{e_{11}}, F_{e_{12}}, \cdots, F_{e_{m n}}\right) \\
= & \bigotimes_{i=1}^{m}\left(\bigotimes_{j=1}^{n} F_{e_{\delta(i) \gamma(j)}}^{\frac{R_{j}}{\sum_{j=1}^{n} R_{j}}}\right)^{\frac{T_{i}}{\sum_{i=1}^{m} T_{i}}},
\end{aligned}
$$

where $R_{1}=1, T_{1}=1 R_{j}=\prod_{l=1}^{j-1} S\left(F_{e_{i \gamma(l)}}\right)$ and $T_{i}=$ $\prod_{k=1}^{i-1} S\left(F_{e_{\delta(k)}}\right) ; S\left(F_{e}\right)$ represents the score function of IFSN $F_{e}$, and $\delta$ and $\gamma$ are permutations of $(1,2, \cdots, m)$ and $(1,2, \cdots, n)$, such that $e_{\delta(i) j} \geq e_{\delta(i-1) j}$ and $e_{i \gamma(j)} \geq e_{i \gamma(j-1)}$ for any $i=2,3, \ldots, m ; j=2,3, \ldots, n$. 
Theorem 4.4. The aggregated value of all IFSNs $F_{e_{i j}}$ by using IFSPOWG operator is still an IFSN defined as follows:

$$
\begin{aligned}
& \operatorname{IFSPOWG}\left(F_{e_{11}}, F_{e_{12}}, \cdots, F_{e_{m n}}\right) \\
& =\left\langle\prod_{i=1}^{m}\left(\prod_{j=1}^{n} \mu_{\delta(i) \gamma(j)}^{\frac{R_{j}}{\sum_{j=1}^{m} R_{j}}}\right)^{\frac{T_{i}}{\sum_{i=1}^{m} T_{i}}},\right. \\
& \left.1-\prod_{i=1}^{m}\left(\prod_{j=1}^{n}\left(1-\nu_{\delta(i) \gamma(j)}\right)^{\frac{R_{j}}{\sum_{j=1}^{n} R_{j}}}\right)^{\frac{T_{i}}{\sum_{i=1}^{m} T_{i}}}\right\rangle
\end{aligned}
$$

where $R_{1}=1, T_{1}=1, R_{j}=\prod_{l=1}^{j-1} S\left(F_{e_{i \gamma(l)}}\right), j=2,3$, $\cdots, n, T_{i}=\prod_{k=1}^{i-1} S\left(F_{e_{\delta(k)}}\right)(i=2,3, \cdots, m)$.

Proof. Proof of this theorem is the same as that of Theorem 4.3.

Example 4.4. Considering Example 4.3 and by using Eq. (1), we get the ordered matrix as follows:

$$
\begin{aligned}
& \gamma\left(e_{1}\right) \quad \gamma\left(e_{2}\right) \quad \gamma\left(e_{3}\right)
\end{aligned}
$$

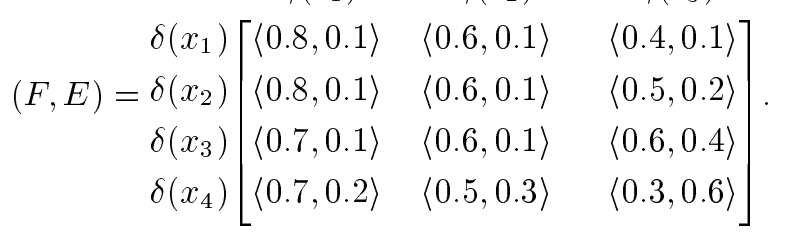

Thus, based on the matrix, values of $R_{j}=$ $\prod_{l=1}^{j-1} S\left(F_{e_{i l}}\right), j=2,3$, and $T_{i}=\prod_{k=1}^{i-1} S\left(F_{e_{k}}\right), i=2,3,4$, are as follows:

$$
R=\left[\begin{array}{lll}
1 & 0.85 & 0.75 \\
1 & 0.85 & 0.75 \\
1 & 0.80 & 0.75 \\
1 & 0.75 & 0.60
\end{array}\right], \quad T=\left[\begin{array}{c}
1 \\
0.7481 \\
0.7529 \\
0.7181
\end{array}\right]
$$

Hence, by Eq. (14), we get:

$$
\begin{aligned}
& \operatorname{IFSPOWG}\left(F_{e_{11}}, F_{e_{12}}, \cdots, F_{e_{43}}\right) \\
& =\left\langle\prod_{i=1}^{4}\left(\prod_{j=1}^{3} \mu_{\delta(i) \gamma(j)}^{\frac{R_{j}}{\sum_{j=1}^{3} R_{j}}}\right)^{\frac{T_{i}}{\sum_{i=1}^{4} T_{i}}},\right. \\
& \left.1-\prod_{i=1}^{4}\left(\prod_{j=1}^{3}\left(1-\nu_{\delta(i) \gamma(j)}\right)^{\frac{R_{j}}{\sum_{j=1}^{3} R_{j}}}\right)^{\frac{T_{i}}{\sum_{i=1}^{4} T_{i}}}\right\rangle \\
& =\langle 0.5927,0.1946\rangle \text {. }
\end{aligned}
$$

As similar to IFSPOWA operator, IFSPOWG operator also satisfies the same properties for the collection of IFSNs $F_{e_{i j}}$.

\section{MCDM based on the proposed operators}

\subsection{An approach based on the proposed operators}

Let $A=\left\{A_{1}, A_{2}, \cdots, A_{t}\right\}$ be the set of alternatives, $E=\left\{e_{1}, e_{2}, \cdots, e_{n}\right\}$ be the set of parameters, and $X=\left\{x_{1}, x_{2}, \cdots, x_{m}\right\}$ be the set of experts giving their preferences corresponding to each alternative $A_{b}$ $(b=1,2, \cdots, t)$ with respect to each parameter $e_{j}$ $(j=1,2, \cdots, n)$ in terms of IFSNs $F_{e_{i j}}=\left\langle\mu_{i j}, \nu_{i j}\right\rangle$. In the following, we develop an approach based on the proposed operator to MCDM with intuitionistic fuzzy soft information, which involves the following steps:

- Step 1: Collect the information related to each alternative under different parameters in terms of intuitionistic fuzzy soft matrix, $D=\left(F_{e_{i j}}\right)=\left\langle\mu_{i j}\right.$, $\left.\nu_{i j}\right\rangle_{m \times n}$

- Step 2: Normalize these collective information decision matrices by transforming the rating values of cost $(C)$ type into benefit $(B)$, if any, by using the normalization formula:

$$
q_{i j}= \begin{cases}F_{e_{i j}}^{c} ; & \text { for cost type parameters } \\ F_{e_{i j}} ; & \text { for benefit type parameters }\end{cases}
$$

where $F_{e_{i j}}^{c}=\left\langle\nu_{i j}, \mu_{i j}\right\rangle$ is the complement of $F_{e_{i j}}=$ $\left\langle\mu_{i j}, \nu_{i j}\right\rangle$;

- Step 3: Calculate $R_{j}(j=1,2, \cdots, n), T_{i}(i=$ $1,2, \cdots, m)$ as follows:

$$
\begin{array}{ll}
T_{1}=1, & R_{1}=1, \\
R_{j}=\prod_{l=1}^{j-1} S\left(F_{e_{i l}}\right), & j=1,2, \cdots, n, \\
T_{i}=\prod_{k=1}^{i-1} S\left(F_{e_{k}}\right), & i=1,2, \cdots, m .
\end{array}
$$

- Step 4: Aggregate IFSNs $q_{i j}^{(b)}(i=1,2, \cdots, m ; j=$ $1,2, \cdots, n)$ for each alternative $A_{b}(b=1,2, \cdots, t)$ into the collective preference value $q^{(b)}$ by the proposed IFSPWA (or IFSPWG, IFSPOWA, IFSPOWG) operator.

- Step 5: By using Eq. (1), we get the score value for each $q^{(b)}(b=1,2, \cdots, t)$;

- Step 6: Rank alternatives $A_{b}(b=1,2, \cdots, t)$ and select the best one(s). 


\subsection{Numerical example}

The above decision-making procedure has been illustrated with a practical example about recruitment of a professor in Mathematics Department for a central Government university. The panel of five experts $x_{1}, x_{2}, x_{3}, x_{4}, x_{5}$ will judge four candidates $A_{1}$, $A_{2}, A_{3}, A_{4}$ and select one candidate on the basis of certain parameters $E=\left\{\right.$ "Qualification $\left(e_{1}\right)$ ", "Teaching experience $\left(e_{2}\right)$ ", "research experience $\left(e_{3}\right)$ ", "number of publications $\left(e_{4}\right)$ ", and "Teaching Ability $\left.\left(e_{5}\right) "\right\}$. Then, we utilize the approach developed to get the most desirable alternative(s).

\subsubsection{By IFSPWA operator}

The steps of the proposed approach have been executed and their detail descriptions are summarized as follows:

- Step 1: The given candidates are being evaluated by five experts to give their grades in terms of IFSNs and are summarized in Tables 2, 3, 4, and 5 , respectively, for each candidate.

- Step 2: Since all the parameters are of the same type, so, there is no need for normalization.

Table 2. Intuitionistic fuzzy soft matrix for the candidate $A_{1}$.

\begin{tabular}{cccccc}
\hline & $\boldsymbol{e}_{\boldsymbol{1}}$ & $\boldsymbol{e}_{\mathbf{2}}$ & $\boldsymbol{e}_{\mathbf{3}}$ & $\boldsymbol{e}_{\mathbf{4}}$ & $\boldsymbol{e}_{\mathbf{5}}$ \\
\hline $\boldsymbol{x}_{\mathbf{1}}$ & $\langle 0.3,0.4\rangle$ & $\langle 0.5,0.1\rangle$ & $\langle 0.6,0.2\rangle$ & $\langle 0.7,0.1\rangle$ & $\langle 0.6,0.2\rangle$ \\
$\boldsymbol{x}_{\mathbf{2}}$ & $\langle 0.6,0.1\rangle$ & $\langle 0.6,0.2\rangle$ & $\langle 0.2,0.4\rangle$ & $\langle 0.5,0.1\rangle$ & $\langle 0.7,0.3\rangle$ \\
$\boldsymbol{x}_{\mathbf{3}}$ & $\langle 0.5,0.1\rangle$ & $\langle 0.7,0.2\rangle$ & $\langle 0.5,0.4\rangle$ & $\langle 0.2,0.2\rangle$ & $\langle 0.4,0.2\rangle$ \\
$\boldsymbol{x}_{\mathbf{4}}$ & $\langle 0.2,0.4\rangle$ & $\langle 0.5,0.1\rangle$ & $\langle 0.6,0.1\rangle$ & $\langle 0.4,0.1\rangle$ & $\langle 0.6,0.2\rangle$ \\
$\boldsymbol{x}_{\mathbf{5}}$ & $\langle 0.6,0.1\rangle$ & $\langle 0.3,0.4\rangle$ & $\langle 0.4,0.3\rangle$ & $\langle 0.6,0.1\rangle$ & $\langle 0.5,0.2\rangle$ \\
\hline
\end{tabular}

Table 3. Intuitionistic fuzzy soft matrix for the candidate $A_{2}$.

\begin{tabular}{cccccc}
\hline & $\boldsymbol{e}_{\boldsymbol{1}}$ & $\boldsymbol{e}_{\boldsymbol{2}}$ & $\boldsymbol{e}_{\mathbf{3}}$ & $\boldsymbol{e}_{\mathbf{4}}$ & $\boldsymbol{e}_{\mathbf{5}}$ \\
\hline $\boldsymbol{x}_{\mathbf{1}}$ & $\langle 0.4,0.3\rangle$ & $\langle 0.5,0.1\rangle$ & $\langle 0.6,0.2\rangle$ & $\langle 0.7,0.1\rangle$ & $\langle 0.7,0.2\rangle$ \\
$\boldsymbol{x}_{\mathbf{2}}$ & $\langle 0.6,0.1\rangle$ & $\langle 0.5,0.3\rangle$ & $\langle 0.4,0.3\rangle$ & $\langle 0.4,0.3\rangle$ & $\langle 0.4,0.1\rangle$ \\
$\boldsymbol{x}_{\mathbf{3}}$ & $\langle 0.5,0.3\rangle$ & $\langle 0.5,0.1\rangle$ & $\langle 0.5,0.3\rangle$ & $\langle 0.3,0.2\rangle$ & $\langle 0.6,0.2\rangle$ \\
$\boldsymbol{x}_{4}$ & $\langle 0.5,0.3\rangle$ & $\langle 0.7,0.3\rangle$ & $\langle 0.4,0.2\rangle$ & $\langle 0.5,0.1\rangle$ & $\langle 0.5,0.2\rangle$ \\
$\boldsymbol{x}_{5}$ & $\langle 0.4,0.2\rangle$ & $\langle 0.5,0.2\rangle$ & $\langle 0.3,0.3\rangle$ & $\langle 0.6,0.1\rangle$ & $\langle 0.4,0.2\rangle$ \\
\hline
\end{tabular}

Table 4. Intuitionistic fuzzy soft matrix for the candidate $A_{3}$.

\begin{tabular}{cccccc}
\hline & $\boldsymbol{e}_{1}$ & $\boldsymbol{e}_{\mathbf{2}}$ & $\boldsymbol{e}_{\mathbf{3}}$ & $\boldsymbol{e}_{4}$ & $\boldsymbol{e}_{\mathbf{5}}$ \\
\hline $\boldsymbol{x}_{\mathbf{1}}$ & $\langle 0.4,0.3\rangle$ & $\langle 0.5,0.4\rangle$ & $\langle 0.5,0.2\rangle$ & $\langle 0.6,0.1\rangle$ & $\langle 0.4,0.2\rangle$ \\
$\boldsymbol{x}_{\mathbf{2}}$ & $\langle 0.5,0.1\rangle$ & $\langle 0.3,0.2\rangle$ & $\langle 0.3,0.2\rangle$ & $\langle 0.4,0.2\rangle$ & $\langle 0.3,0.2\rangle$ \\
$\boldsymbol{x}_{\mathbf{3}}$ & $\langle 0.5,0.3\rangle$ & $\langle 0.5,0.1\rangle$ & $\langle 0.4,0.2\rangle$ & $\langle 0.2,0.2\rangle$ & $\langle 0.5,0.4\rangle$ \\
$\boldsymbol{x}_{\mathbf{4}}$ & $\langle 0.5,0.1\rangle$ & $\langle 0.4,0.5\rangle$ & $\langle 0.3,0.2\rangle$ & $\langle 0.7,0.2\rangle$ & $\langle 0.3,0.2\rangle$ \\
$\boldsymbol{x}_{\mathbf{5}}$ & $\langle 0.7,0.1\rangle$ & $\langle 0.4,0.6\rangle$ & $\langle 0.4,0.2\rangle$ & $\langle 0.3,0.1\rangle$ & $\langle 0.6,0.1\rangle$ \\
\hline
\end{tabular}

Table 5. Intuitionistic fuzzy soft matrix for the candidate $A_{4}$.

\begin{tabular}{cccccc}
\hline & $\boldsymbol{e}_{\mathbf{1}}$ & $\boldsymbol{e}_{\mathbf{2}}$ & $\boldsymbol{e}_{\mathbf{3}}$ & $\boldsymbol{e}_{\mathbf{4}}$ & $\boldsymbol{e}_{\mathbf{5}}$ \\
\hline $\boldsymbol{x}_{\mathbf{1}}$ & $\langle 0.3,0.4\rangle$ & $\langle 0.8,0.1\rangle$ & $\langle 0.7,0.1\rangle$ & $\langle 0.4,0.3\rangle$ & $\langle 0.2,0.3\rangle$ \\
$\boldsymbol{x}_{\mathbf{2}}$ & $\langle 0.5,0.1\rangle$ & $\langle 0.4,0.2\rangle$ & $\langle 0.4,0.2\rangle$ & $\langle 0.6,0.1\rangle$ & $\langle 0.2,0.6\rangle$ \\
$\boldsymbol{x}_{\boldsymbol{3}}$ & $\langle 0.2,0.1\rangle$ & $\langle 0.4,0.2\rangle$ & $\langle 0.5,0.4\rangle$ & $\langle 0.4,0.2\rangle$ & $\langle 0.5,0.2\rangle$ \\
$\boldsymbol{x}_{\mathbf{4}}$ & $\langle 0.7,0.2\rangle$ & $\langle 0.5,0.1\rangle$ & $\langle 0.6,0.1\rangle$ & $\langle 0.4,0.1\rangle$ & $\langle 0.7,0.1\rangle$ \\
$\boldsymbol{x}_{\boldsymbol{5}}$ & $\langle 0.5,0.2\rangle$ & $\langle 0.5,0.4\rangle$ & $\langle 0.4,0.2\rangle$ & $\langle 0.3,0.2\rangle$ & $\langle 0.7,0.1\rangle$ \\
\hline
\end{tabular}

- Step 3: Utilize Eqs. (15)-(17) to find $R_{j}^{(b)}$ and $T_{i}^{(b)}$ ( $b=1,2,3,4)$ corresponding to each candidate $A_{1}$, $A_{2}, A_{3}$ and $A_{4}$, then we get:

$$
R_{j}^{(1)}=\left[\begin{array}{lllll}
1 & 0.45 & 0.70 & 0.70 & 0.80 \\
1 & 0.75 & 0.70 & 0.40 & 0.70 \\
1 & 0.70 & 0.75 & 0.55 & 0.50 \\
1 & 0.40 & 0.70 & 0.75 & 0.65 \\
1 & 0.75 & 0.45 & 0.55 & 0.75
\end{array}\right] \text {, }
$$$$
R_{j}^{(2)}=\left[\begin{array}{lllll}
1 & 0.55 & 0.70 & 0.70 & 0.80 \\
1 & 0.75 & 0.60 & 0.55 & 0.55 \\
1 & 0.60 & 0.70 & 0.60 & 0.55 \\
1 & 0.60 & 0.70 & 0.60 & 0.70 \\
1 & 0.60 & 0.65 & 0.50 & 0.75
\end{array}\right] \text {, }
$$$$
R_{j}^{(3)}=\left[\begin{array}{ccccc}
1 & 0.55 & 0.55 & 0.65 & 0.75 \\
1 & 0.70 & 0.55 & 0.55 & 0.60 \\
1 & 0.60 & 0.70 & 0.60 & 0.50 \\
1 & 0.70 & 0.45 & 0.55 & 0.75 \\
1 & 0.80 & 0.40 & 0.60 & 0.60
\end{array}\right]
$$$$
R_{j}^{(4)}=\left[\begin{array}{ccccc}
1 & 0.45 & 0.85 & 0.80 & 0.55 \\
1 & 0.70 & 0.60 & 0.60 & 0.75 \\
1 & 0.55 & 0.60 & 0.55 & 0.60 \\
1 & 0.75 & 0.70 & 0.75 & 0.65 \\
1 & 0.65 & 0.55 & 0.60 & 0.55
\end{array}\right]
$$$$
T_{i}^{(1)}=\left[\begin{array}{c}
1 \\
0.6760 \\
0.6834 \\
0.6554 \\
0.6427
\end{array}\right], \quad T_{i}^{(2)}=\left[\begin{array}{c}
1 \\
0.7063 \\
0.6521 \\
0.6360 \\
0.6554
\end{array}\right] \text {, }
$$

$$
T_{i}^{(3)}=\left[\begin{array}{c}
1 \\
0.6272 \\
0.6092 \\
0.6076 \\
0.6327
\end{array}\right], \quad T_{i}^{(4)}=\left[\begin{array}{c}
1 \\
0.6474 \\
0.6243 \\
0.6017 \\
0.7403
\end{array}\right]
$$


- Step 4: Based on these values, the different preferences of the alternatives are aggregated into collective one $\left(q^{(b)}\right)$ for each $b$ th candidate by using Eq. (4); so, we get:

$$
\begin{array}{ll}
q^{(1)}=\langle 0.5166,0.1853\rangle, & q^{(2)}=\langle 0.5157,0.1942\rangle, \\
q^{(3)}=\langle 0.4614,0.1943\rangle, & q^{(4)}=\langle 0.4932,0.1839\rangle .
\end{array}
$$

- Step 5: The score values corresponding to each candidate are:

$$
\begin{aligned}
& S\left(q^{(1)}\right)=0.6657, \quad S\left(q^{(2)}\right)=0.6608, \\
& S\left(q^{(3)}\right)=0.6335, \quad S\left(q^{(4)}\right)=0.6547 .
\end{aligned}
$$

- Step 6: Thus, the ranking is $S\left(q^{(1)}\right)>S\left(q^{(2)}\right)>$ $S\left(q^{(4)}\right)>S\left(q^{(3)}\right)$; hence, $A_{1}$ is the best candidate for the required post.

\subsubsection{By IFSPWG operator}

Based on IFSPWG operator, the following steps have been performed.

- Step 1: Same as that of above.

- Step 2: All the parameters are of the same type, so there is no need of normalizing the data.

- Step 3: Eqs. (15)-(17) are utilized to find $T_{i}^{(b)}(b=$ $1,2,3,4)$ corresponding to each candidate, and then we get:

$$
T_{i}^{(1)}=\left[\begin{array}{c}
1 \\
0.6335 \\
0.6296 \\
0.6132 \\
0.5889
\end{array}\right], \quad T_{i}^{(2)}=\left[\begin{array}{c}
1 \\
0.6803 \\
0.6280 \\
0.6178 \\
0.6376
\end{array}\right],
$$

$$
T_{i}^{(3)}=\left[\begin{array}{c}
1 \\
0.6075 \\
0.5967 \\
0.5795 \\
0.5873
\end{array}\right], \quad T_{i}^{(4)}=\left[\begin{array}{c}
1 \\
0.5736 \\
0.5624 \\
0.5692 \\
0.7223
\end{array}\right] .
$$

- Step 4: Based on these equations, the aggregated values obtained by using Eq. (4) for each candidate are:

$$
\begin{array}{ll}
q^{(1)}=\langle 0.4632,0.2251\rangle, & q^{(2)}=\langle 0.4897,0.2152\rangle, \\
q^{(3)}=\langle 0.4295,0.2378\rangle, & q^{(4)}=\langle 0.4319,0.2285\rangle .
\end{array}
$$

- Step 5: The score values corresponding to each candidate are:

$$
\begin{aligned}
& S\left(q^{(1)}\right)=0.6191, \quad S\left(q^{(2)}\right)=0.6372, \\
& S\left(q^{(3)}\right)=0.5958, \quad S\left(q^{(4)}\right)=0.6017 .
\end{aligned}
$$

- Step 6: Therefore, the ranking is $S\left(q^{(2)}\right)>$ $S\left(q^{(1)}\right)>S\left(q^{(4)}\right)>S\left(q^{(3)}\right)$; hence, $A_{2}$ is the best candidate for the required post.

\subsection{Comparative studies}

To demonstrate the effectiveness of the proposed approach as compared to the existing ones for MCDM, an analysis has been conducted by using different operators as proposed by various researchers $[5-8,12]$. So, the grades corresponding to different parameters of each candidate are aggregated by geometric operator corresponding to the weight vector $(0.2,0.2,0.2,0.2,0.2)^{T}$ and their aggregated results are summarized in Table 6 . By using these aggregated values, the different approaches as proposed by various authors [5-8,12] have been applied to it and their score values as well as ranking of each candidate are computed and tabulated in Table 7.

Table 6. Aggregated intuitionistic fuzzy soft matrix for candidates.

\begin{tabular}{ccccc}
\hline & $\boldsymbol{A}_{\mathbf{1}}$ & $\boldsymbol{A}_{\mathbf{2}}$ & $\boldsymbol{A}_{\mathbf{3}}$ & $\boldsymbol{A}_{\mathbf{4}}$ \\
\hline $\boldsymbol{x}_{\mathbf{1}}$ & $\langle 0.4807,0.2150\rangle$ & $\langle 0.5368,0.1841\rangle$ & $\langle 0.4554,0.2921\rangle$ & $\langle 0.4103,0.2552\rangle$ \\
$\boldsymbol{x}_{\mathbf{2}}$ & $\langle 0.5796,0.2052\rangle$ & $\langle 0.4786,0.2063\rangle$ & $\langle 0.3508,0.1761\rangle$ & $\langle 0.3704,0.2990\rangle$ \\
$\boldsymbol{x}_{\boldsymbol{3}}$ & $\langle 0.4854,0.1879\rangle$ & $\langle 0.4973,0.1991\rangle$ & $\langle 0.4512,0.2497\rangle$ & $\langle 0.3596,0.1879\rangle$ \\
$\boldsymbol{x}_{\boldsymbol{4}}$ & $\langle 0.4107,0.2104\rangle$ & $\langle 0.5562,0.2528\rangle$ & $\langle 0.4103,0.3011\rangle$ & $\langle 0.5839,0.1261\rangle$ \\
$\boldsymbol{x}_{\mathbf{5}}$ & $\langle 0.4407,0.2512\rangle$ & $\langle 0.4440,0.1959\rangle$ & $\langle 0.4947,0.3264\rangle$ & $\langle 0.5111,0.2550\rangle$ \\
\hline
\end{tabular}

Table 7. Comparative studies with some of the existing approaches.

\begin{tabular}{lccccc}
\hline \multirow{2}{*}{ Method } & \multicolumn{4}{c}{ Score } & \multirow{2}{*}{ Ranking } \\
\cline { 2 - 4 } & $\boldsymbol{s}\left(\boldsymbol{q}^{(\mathbf{1})}\right)$ & $\boldsymbol{s}\left(\boldsymbol{q}^{(\mathbf{2})}\right)$ & $\boldsymbol{s}\left(\boldsymbol{q}^{(\mathbf{3})}\right)$ & $\boldsymbol{s}\left(\boldsymbol{q}^{(4)}\right)$ & \\
\hline Xu [5] & 0.1271 & 0.1443 & 0.0951 & 0.1175 & $q_{2} \succ q_{1} \succ q_{4} \succ q_{3}$ \\
Xu and Yager [6] & 0.1255 & 0.1411 & 0.0920 & 0.1086 & $q_{2} \succ q_{1} \succ q_{4} \succ q_{3}$ \\
Wang and Liu [7] & 0.1257 & 0.1416 & 0.0925 & 0.1098 & $q_{2} \succ q_{1} \succ q_{4} \succ q_{3}$ \\
Wang and Liu [8] & 0.1269 & 0.1438 & 0.0946 & 0.1162 & $q_{2} \succ q_{1} \succ q_{4} \succ q_{3}$ \\
Verma and Sharma [12] & 0.6275 & 0.6435 & 0.5957 & 0.6144 & $q_{2} \succ q_{1} \succ q_{4} \succ q_{3}$ \\
\hline
\end{tabular}




\section{Conclusion}

The aim of this paper is to present the intuitionistic fuzzy soft prioritized aggregation operator to solve MCDM problem in which the priority level for each parameter and expert is different. For that matter, a series of prioritized averaging/geometric aggregation operators, such as IFSPWA, IFSPOWA, IFSPWG, and IFSPOWG, have been presented. The important characteristic of these operators is that they take the parameters and decision-makers according to their priority level. Finally, an approach to solving MCDM problems under the intuitionistic fuzzy soft set environment has been given. To demonstrate the proposed work, a practical example about the recruitment of the candidate is given, and the results obtained by the proposed approach are compared with those of the existing methods. In the future work, we shall apply these operators to other fields such as mathematical programming, cluster analysis, big-data analysis, and so on.

\section{Acknowledgments}

The first author (Rishu Arora) would like to thank the Department of Science \& Technology, New Delhi, India for providing financial support under WOS-A scheme wide file no. SR/WOS-A/PM-77/2016 for the preparation of this manuscript. The second author (Harish Garg) would like to thanks the Thapar Institute of Engineering \& Technology (Deemed University) Patiala for providing the financial support under SEED Money Grant wide letter no. TU/DORSP/57/1910.

\section{References}

1. Garg, H., Agarwal, N. and Tripathi, A. "Some improved interactive aggregation operators under interval-valued intuitionistic fuzzy environment and its application to decision making process", Scientia Iranica, Transactions E: Industrial Engineering, 24(5), pp. 2581-2604 (2017).

2. Zadeh, L.A. "Fuzzy sets", Inform. and Control, 8, pp. 338-353 (1965).

3. Attanassov, K.T. "Intuitionistic fuzzy sets", Fuzzy Sets and Systems, 20, pp. 87-96 (1986).

4. Atanassov, K. and Gargov, G. "Interval-valued intuitionistic fuzzy sets", Fuzzy Sets and Systems, 31, pp. 343-349 (1989).

5. Xu, Z.S. "Intuitionistic fuzzy aggregation operators" IEEE Trans. of Fuzzy System, 15, pp. 1179-1187 (2007).

6. Xu, Z.S. and Yager, R.R. "Some geometric aggregation operators based on intuitionistic fuzzy sets", Int. J. Gen. Syst., 35, pp. 417-433 (2006).
7. Wang, W.Z. and Liu, X.W. "Intuitionistic fuzzy geometric aggregation operators based on Einstein operations", Int. J. of Intell. Syst, 26, pp. 1049-1075 (2011).

8. Wang, W. and Liu, X. "Intuitionistic fuzzy information aggregation using Einstein operations", IEEE Trans. Fuzzy Systems, 20(5), pp. 923-938 (2012).

9. Garg, H. "Generalized intuitionistic fuzzy interactive geometric interaction operators using Einstein t-norm and t-conorm and their application to decision making", Comput. Ind. Eng, 101, pp. 53-69 (2016).

10. Garg, H., Agarwal, N. and Tripathi, A. "Entropy based multi-criteria decision making method under fuzzy environment and unknown attribute weights", Global Journal of Technology and Optimization, 6, pp. 13-20 (2015).

11. Garg, H. "A new generalized improved score function of interval-valued intuitionistic fuzzy sets and applications in expert systems", Appl. Soft Comput., 38, pp. 988-999 (2016).

12. Verma, R. and Sharma, B. "Intuitionistic fuzzy Einstein prioritized weighted average operators and their application to multiple attribute group decision making", Applied Mathematics \& Information Sciences, 9(6), pp. 3095-3107 (2015).

13. Xu, Z. and Chen, J. "Approach to group decision making based on interval valued intuitionistic judgment matrices", Systems Engineering - Theory and Practice, 27(4), pp. 126-133 (2007).

14. Xu, Z.S. "Methods for aggregating interval-valued intuitionistic fuzzy information and their application to decision making", Control and Decision, 22(2), pp. 215-219 (2007).

15. Wei, G. "Some induced geometric aggregation operators with intuitionistic fuzzy information and their application to group decision making", Appl. Soft Comput., 10, pp. 423-431 (2010).

16. Garg, H. "A novel accuracy function under intervalvalued pythagorean fuzzy environment for solving multicriteria decision making problem", Journal of Intelligent and Fuzzy Systems, 31(1), pp. 529-540 (2016).

17. Garg, H. "A novel correlation coefficients between pythagorean fuzzy sets and its applications to decisionmaking processes", Inter. Intell. Syst, 31(12), pp. 1234-1253 (2016).

18. Garg, H. "A new generalized pythagorean fuzzy information aggregation using Einstein operations and its application to decision making", Inter. Intell. Syst, 31(9), pp. 886-920 (2016).

19. Garg, H. "Generalized intuitionistic fuzzy multiplicative interactive geometric operators and their application to multiple criteria decision making", Inter. J. of Mach. Learn. Cybernet, 7(6), pp. 1075-1092 (2016).

20. Wei, G.W. and Merigo, J.M. "Methods for strategic decision-making problems with immediate probabili- 
ties in intuitionistic fuzzy setting", Scientia Iranica, 19(6), pp. 1936-1946 (2012).

21. Garg, H. "Some series of intuitionistic fuzzy interactive averaging aggregation operators", SpringerPlus, 5(1), pp. 1-27 (2016). DOI: 10.1186/s40064-016-2591-9

22. Wei, G.W. "Some arithmetic aggregation operators with intuitionistic trapezoidal fuzzy numbers and their application to group decision making", Journal Comput., 5(3), pp. 345-351 (2010).

23. Wei, G. and Zhao, X. "An approach to multiple attribute decision making with combined weight information in interval-valued intuitionistic fuzzy environmental", Control Cybernet., 41(1), pp. 97-112 (2012).

24. Molodtsov, D. "Soft set theory - first results", Computer and Mathematics with Applications, 27(4-5), pp. 19-31 (1999).

25. Maji, P.K., Biswas, R. and Roy, A.R. "Soft set theory", Computers and Mathematics with Applications, 45(45), pp. 555-562 (2003).

26. Ali, M., Feng, F., Liu, X., Min, W. and Shabir, M. "On some new operations in soft set theory", Computer and Mathematics with Applications, 57(9), pp. 1547-1553 (2009).

27. Maji, P.K., Biswas, R. and Roy, A.R. "Fuzzy soft sets", Journal Fuzzy Math., 9(3), pp. 589-602 (2001).

28. Maji, P.K., Biswas, R. and Roy, A. "Intuitionistic fuzzy soft sets", Journal Fuzzy Math., 9(3), pp. 677692 (2001).

29. Cagman, N. and Deli, I. "Intuitionistic fuzzy parameterized soft set theory and its decision making", Appl. Soft. Comput., 28, pp. 109-113 (2015).

30. Alkhazaleh, S. and Salleh, A.R. "Fuzzy soft expert set and its application", Appl. Math., 5, pp. 1349-1368 (2014).

31. Yang, H.L. "Notes on generalized fuzzy soft sets", Journal of Mathematical Research and Exposition, 31(3), pp. 567-570 (2011).

32. Majumdar, P. and Samanta, S.K. "Generalized fuzzy soft sets", Computers and Mathematics with Applications, 59(4), pp. 1425-1432 (2010).

33. Agarwal, M., Biswas, K.K. and Hanmandlu, M. "Generalized intuitionistic fuzzy soft sets with applications in decision-making", Appl. Soft Comput., 13, pp. 35523566 (2013).

34. Majumdar, P. and Samanta, S. "Similarity measure of soft sets", New Math. Nat. Comput, 4(1), pp. 1-12 (2008).

35. Feng, Q. and Zheng, W. "New similarity measures of fuzzy soft sets based on distance measures", Ann. Fuzzy Math. Inform., 7(4), pp. 669-686 (2014).

36. Mukherjee, A. and Sarkar, S. "Similarity measures for interval-valued intuitionistic fuzzy soft sets and its application in medical diagnosis problem", New Trends Math. Sci., 2(3), pp. 159-165 (2014).
37. Khalid, A. and Abbas, M. "Distance measures and operations in intuitionistic and interval-valued intuitionistic fuzzy soft set theory", Int. J. Fuzzy Syst., 17(3), pp. 490-497 (2015).

38. Liu, Z., Qin, K. and Pei, Z. "Similarity measure and entropy of fuzzy soft sets", The Scientific World Journal, 2014 Article ID 161607, 10 pages (2014).

39. Garg, H., Agarwal, N. and Tripathi, A. "Fuzzy number intuitionistic fuzzy soft sets and its properties", Journal of Fuzzy Set Valued Analysis, 2016(3), pp. 196-213 (2016).

40. Feng, F., Juna, Y.B., Liu, X. and Li, L. "An adjustable approach to fuzzy soft set based decision making", $J$. Comput. Appl. Math., 234(1), pp. 10-20 (2010).

41. Peng, X. and Yang, Y. "Algorithms for interval-valued fuzzy soft sets in stochastic multi-criteria decision making based on regret theory and prospect theory with combined weight", Appl. Soft Comput, 54, pp. 415-430 (2017).

42. Yager, R.R. "Prioritized aggregation operators", Internat. J. Approx. Reason., 48(1), pp. 263-274, (2008).

43. Roy, A.R. and Maji, P.K. "A fuzzy soft set theoretic approach to decision making problems", J. Comput. Appl. Math., 203(2), pp. 412-418 (2007).

\section{Biographies}

Rishu Arora received the Master degree in Mathematics from Department of Mathematics, Guru Nanak Dev University, Amritsar, India, in 2010. Presently, she is working towards her $\mathrm{PhD}$ degree in Thapar Institute of Engineering \& Technology (Deemed University) Patiala, Punjab, India. She has more than three years of teaching experience in different subjects of Engineering Mathematics. She has qualified GATE in 2012-2015. Her current research interests are in the areas of multi criteria decision-making, aggregation operator, intuitionistic fuzzy soft set. She has published 5 papers in the international reputed SCI journals.

Harish Garg is an Assistant Professor at Thapar Institute of Engineering \& Technology (Deemed University), Patiala, India. Prior to joining this University, Dr. Garg received a PhD in Applied Mathematics with specialization of reliability theory and soft computing techniques from Indian Institute of Technology Roorkee, India, in 2013; he also received an MSc in Mathematics from Punjabi University, Patiala, India, in 2008. His research interests are in the field of computational intelligence, multi-criteria decision-making problems, reliability theory, optimization techniques, various nature-inspired algorithms (e.g., genetic algorithms, swarm optimization), fuzzy and intuitionistic fuzzy set theory, expert systems. Application areas include a wide range of industrial and structural 
engineering design problems. Garg has authored/coauthored over 120 technical papers published in the refereed International Journals. He has published six book chapters. He also serves as an Editorial Board member of various International Journals. He is listed in International Who's Who of Professionals, Marquis Who's Who in the World, and Marquis Who's Who in Science and Engineering. In the year 2016 and 2017, Dr. Garg was privileged an awarded outstanding reviewer for the journal Applied Soft Computing, Applied Mathematical Modeling and Engineering Applications of Artificial Intelligence, Elsevier. His Google citations are over 1550. For more details, visit http://sites.google.com/site/harishg58iitr/ 